NISSUNA UMANA INVESTIGAZIONE SI PUO DIMANDARE VERA SCIENZIA S'ESSA NON PASSA PER LE MATEMATICHE DIMOSTRAZIONI LEONARDO DA VINCI

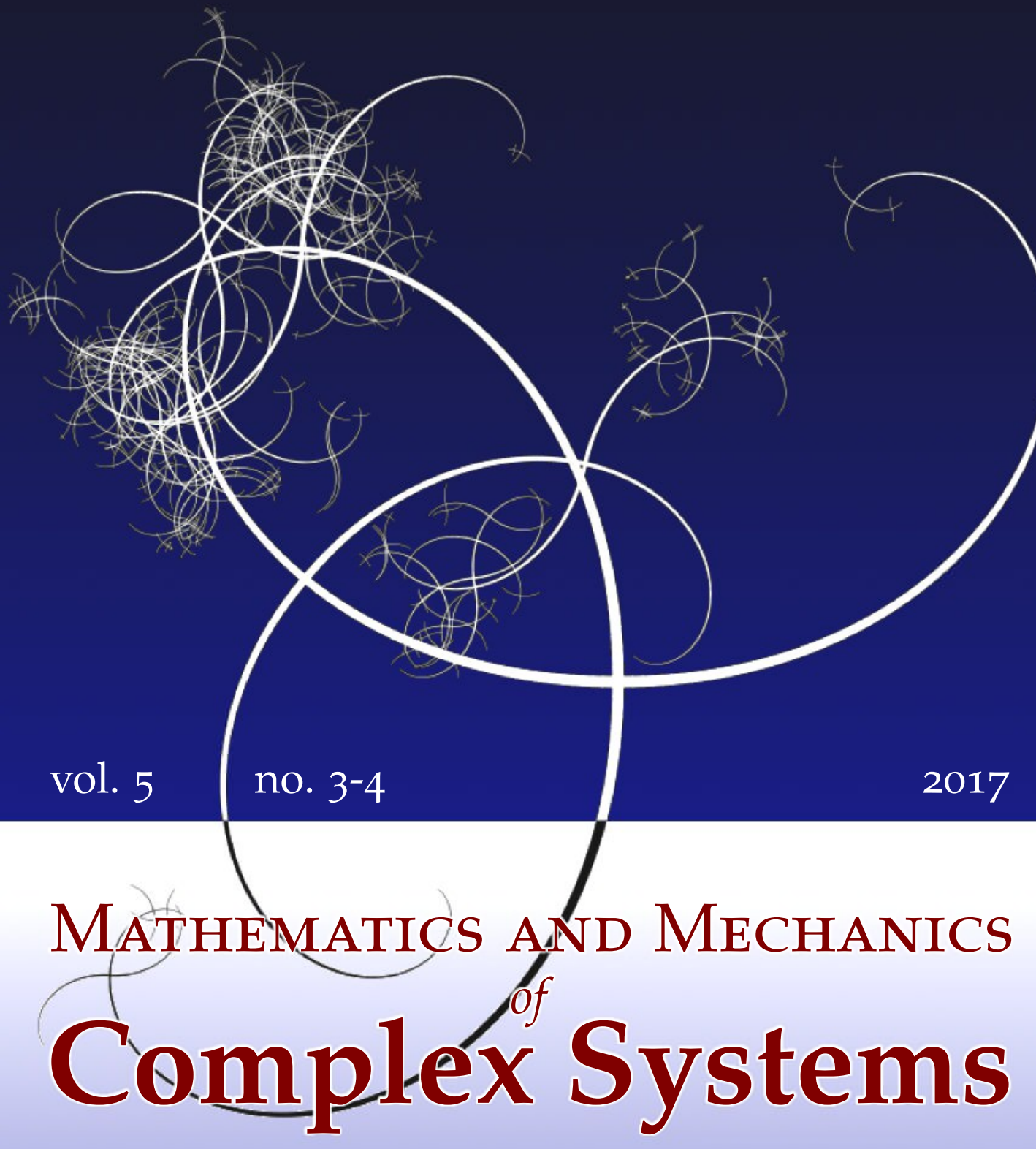

Simon R. Eugster AND CHRistoph Glocker ON THE NOTION OF STRESS IN CLASSICAL CONTINUUM MECHANICS 


\title{
ON THE NOTION OF STRESS IN CLASSICAL CONTINUUM MECHANICS
}

\author{
Simon R. Eugster AND Christoph Glocker
}

\begin{abstract}
A variational formulation of continuum mechanics, in which the principle of virtual work and the variational law of interaction are postulated as the basic axioms, is still controversially discussed. In particular, not widely accepted is the internal virtual work contribution of a continuum, as postulated as a smooth density integrated over the deformed configuration of the body, in which the stress field is defined as the quantity dual to the gradient of the virtual displacement field. The question arises whether this internal virtual work can be deduced, rather than just postulated, from already known mechanical concepts completely within the variational framework. To achieve such a derivation, we give in this paper an interpretation of Piola's micro-macro identification procedure in view of the Riemann integral, which naturally provides in its mathematical definition a micro-macro relation between the discrete system of infinitesimal volume elements and the continuum. Accordingly, we propose a definition of stress on the micro level of the infinitesimal volume elements. In particular, the stress is defined as the internal force effects of the body that model the mutual force interaction between neighboring infinitesimal volume elements. The internal virtual work of the continuum is then obtained by Piola's micro-macro identification procedure, where in the limit of vanishing volume elements the virtual work of the continuous macromodel is identified with the virtual work of the discrete micromodel. In the course of this procedure, the stress tensor emerges directly as the quantity dual to the gradient of the virtual displacement field. Furthermore, we try to gather important results of variational continuum mechanics, which have appeared here and there in very diverse forms, in order to underline once more the strength of a variational formulation of continuum mechanics.
\end{abstract}

\section{Introduction}

To date, there are essentially two different ways to postulate the foundations of continuum mechanics. The first method, henceforth called the nonvariational approach, was conceived mainly by Cauchy $[1823 ; 1827 \mathrm{a}]$ and assumes forces and

\section{Communicated by Francesco dell'Isola.}

MSC2010: 70A05, 74A10.

Keywords: continuum mechanics, virtual work, law of interaction, stress, micro-macro identification. 
moments as the elemental quantities. The second method, which traces back to Lagrange [1788] and Piola [1832; 1848; 2014; dell' Isola et al. 2015a], is of a variational nature and defines forces in a generalized sense as the quantities dual to the virtual displacement field and the gradients thereof. The variational approach has gained much attention during the last half century, especially in the field of generalized continua like gradient materials and polar and micromorphic media; see for instance [dell'Isola et al. 2015b; 2011; Toupin 1964; Germain 1973b]. Most of these generalized theories cannot be stated straightforwardly by using the nonvariational approach. For an evaluative comparison of both the variational and the nonvariational formulations of continuum mechanics and for further references on this topic, we refer to [dell'Isola et al. 2017] and works cited therein. In what follows and without claiming to be in any way complete, we try to review the vast amount of literature on the various formulations of classical continuum mechanics that has appeared in the last two centuries. Since we focus in this treatise on the notion of stress, we omit the discussion about inertia forces. As a consequence, we refer exclusively to the terminology used in statics, e.g., the equilibrium of forces and moments, instead of addressing the balance of linear and angular momentum.

The first formulation of continuum mechanics can be attributed to Cauchy with his celebrated publications [Cauchy $1823 ; 1827 \mathrm{a}$ ]. Cauchy restricted forces to be of volume and surface nature only. He assumed the force interaction between an arbitrary subbody and the rest of the body to take place exclusively by surface forces, called stress vectors. This requirement is nowadays referred to as the stress principle of Euler and Cauchy [Truesdell and Toupin 1960, §200]. Cauchy implicitly made the assumption, known as Cauchy's postulate, that a stress vector for a given body point depends only on the normal vector to an imagined cutting or contact surface passing through this point. By postulating the equilibrium of forces at an infinitesimal parallelepiped, he proved that the stress vectors acting upon opposite sides of the same surface at a given point are equal in magnitude and opposite in direction, which is referred to as Cauchy's lemma. Applying then the equilibrium of forces at an infinitesimal tetrahedron, Cauchy's stress theorem shows that the stress vector for a body point depends linearly on the normal vector of the imagined contact surface. Consequently, Cauchy's stress theorem asserts the existence of the stress tensor field [Truesdell 1991, pp. 174-175]. Furthermore, by postulating the equilibrium of moments at an infinitesimal parallelepiped, Cauchy proved the symmetry of the stress tensor. ${ }^{1}$ Note that in all proofs boundedness assumptions on the applied external forces are involved. In a further publication,

\footnotetext{
${ }^{1}$ Cauchy's lemma and the symmetry of the stress tensor are formulated in [Cauchy 1827a] as "Théorème I" and "Théorème II", respectively. The celebrated stress theorem of Cauchy has to be extracted out of the text and the formulas on [Cauchy 1827a, pp. 68-69].
} 
Cauchy [1827b] derived from the equilibrium of forces at an infinitesimal parallelepiped together with the results of the stress theorem the local equilibrium equations. Poisson [1829, §11] as well as Thomson and Tait [1867, §698] showed by integrating the local equilibrium equations over a finite volume and by applying the divergence theorem that these equations are the sufficient as well as necessary conditions for the equilibrium of the body. This is, the equilibrium of forces and moments in integral form has to hold for the body and all its subbodies. Lamé $[1852, \S 32]$ and Clebsch $[1862, \S 16]$ derived the (virtual) work contribution of the body. They attained the (virtual) work of the body by multiplying the local equilibrium equations with infinitesimal (virtual) displacements, followed by an integration over the body and application of the divergence theorem, and final usage of the surface boundary conditions.

Kirchhoff [1876, Vorlesung 11] reversed the direction of argumentation and based continuum mechanics on the equilibrium of forces and moments in integral form [Müller and Timpe 1914, p. 23]. Kirchhoff proposed, since every subbody is again a body, that the integral balance laws not only hold for the body, but also for every subbody. By using the results of Cauchy's stress theorem in the integral balance laws and by applying the divergence theorem, the local equilibrium equations as well as the symmetry of the stress tensor can be extracted by a localization argument. Also the axiomatic scheme of Noll [1959] followed the approach of Kirchhoff and highly influenced the celebrated work of Truesdell and Toupin [1960, §196]. As a consequence of the popularity of [Truesdell and Toupin 1960], this approach has become standard in continuum mechanics and can be found in a wealth of modern textbooks [Altenbach and Altenbach 1994; Başar and Weichert 2000; Becker and Bürger 1975; Bertram 2012; Chadwick 1999; Ciarlet 1988; Dvorkin and Goldschmit 2006; Eringen 1980; Gurtin 1981; Haupt 2002; Holzapfel 2000; Liu 2002; Malvern 1965; Ogden 1997; Sedov 1972; Spencer 2004; Truesdell and Noll 1965]. Furthermore, Noll [1974] showed that the balance laws can be derived from the objectivity of the work done by the applied (external) forces and postulated this objectivity as a fundamental axiom of continuum mechanics; see also [Truesdell 1991].

An axiomatization of continuum mechanics at an earlier date and the attempt to partially solve the sixth problem of Hilbert ${ }^{2}$ has been given by Hamel in [1912, $\S 38-39$ ] or [1927, §II.b. $\alpha$ ], whose approach differs from Cauchy's in only one point: instead of claiming the equilibrium of moments at an infinitesimal volume

\footnotetext{
${ }^{2}$ Hilbert's sixth problem was proposed in a lecture at the international congress of mathematicians at Paris in 1900 [1901; 1902] "to treat in the same manner, by means of axioms, those physical sciences in which mathematics plays an important part; in the first rank are the theory of probabilities and mechanics."
} 
element, Hamel demanded the stress tensor be symmetric, which he denoted the Boltzmann axiom.

Cauchy's theory of continuum mechanics is based on the insights of Newton and Euler, where forces and moments are the primitives of mechanics. In contrast, Piola was highly influenced by Lagrange's Mécanique Analytique and the calculus of variation (see the Introduction of [Piola 1832] ${ }^{3}$ ). He understood forces in the sense of duality as linear functionals on virtual displacements. While the theories developed in [Piola 1832] are for rigid bodies only, Piola [1848] formulated his variational theory of mechanics also for deformable bodies, with the principle of virtual work as the fundamental equation. Piola introduced between the reference and the deformed configurations an intermediate configuration which differs from the deformed configuration by only a rigid body motion. The stresses are defined as indeterminate Lagrange multipliers to the rigidity constraints between the intermediate and deformed configuration [Piola 2014, Chapter 1, pp. 83-84]. By claiming the principle of virtual work with its corresponding contributions, Piola obtained by an appropriate substitution of variables and integration by parts the local equilibrium equations of Cauchy and the corresponding force boundary conditions. In fact, Piola introduced the (Cauchy) stress tensor as a linear functional on the symmetrized gradient of the virtual displacement field. As a direct consequence of this definition, which already contains objectivity assumptions, the stress tensor is symmetric. Furthermore, Piola derived the integral balance laws for an arbitrary subbody by choosing virtual displacement fields which respect the rigidity constraint between the intermediate and the deformed configurations [Piola 2014, Chapter 1, pp. 85-86]. A very similar approach, without reference to Piola, can be found in [Hamel 1967, Chapter III, §6]. The variational theory of Piola has been made even clearer by the encyclopedia article [Hellinger 1914]. Hellinger in his theory defined forces and stresses as linear forms on the virtual displacement field and the gradient thereof, and proposed the principle of virtual work as the fundamental equation in continuum mechanics. The gradient of the virtual displacement field does not imply a symmetric stress tensor. Hellinger [1914, p. 619] mentioned therefore that the symmetry follows either from the Boltzmann axiom of Hamel or from the equilibrium of moments in integral form. An exegetic series about [Hellinger 1914] including the complete translation into English has just been finished by Eugster and dell'Isola [2017a; 2017b; 2017c].

After the variational formulation of continuum mechanics was almost buried into oblivion, there has been a renaissance of the variational theory in the 1970s by the publications of Germain [1972; 1973a; 1973b]; see also [Maugin 1980]. Therein, first and second gradient theories as well as theories for continua with

\footnotetext{
${ }^{3}$ An English translation is given in [Capecchi and Ruta 2015, pp. 90-93].

${ }^{4}$ An English translation is given in [Piola 2014].
} 
microstructure have been formulated by defining virtual work contributions in the sense of duality and by the postulation of the principle of virtual work as the basic axiom. Maybe the most important contribution, which varies from Piola and Hellinger, is the explicit statement of the axiom of power of internal forces. This axiom demands the virtual work of internal forces to vanish for all rigid virtual displacements and induces the symmetry of the stress in classical continuum mechanics. To date, textbooks on continuum mechanics based on the variational approach can exclusively be found in France, i.e., [Germain 1986; Lemaitre and Chaboche 1990; Salençon 2000; 2001]. Following the ideas of Germain [1972], dell'Isola et al. [2015b; 2011; 2012] obtain from the theory of distributions by L. Schwartz [1951] a representation of the virtual work in terms of $N$-th order stresses which are defined as the quantities dual to the $N$-th gradients of the virtual displacement field. A similar representation of forces in a continuum has already been proposed by Segev [1986]. In this generalized theory, the classical continuum is embedded and obtained for $N=1$. Consequently, the theory of the classical continuum is also referred to as first gradient theory. Another axiomatization of variational nature, also for an $N$-th gradient continuum, is from Bertram [1989] and Bertram and Forest [2007]. They base continuum mechanics on invariance requirements upon a general principle of virtual power, as a linear and continuous extension of the balance of work. In contrast to [Germain 1972], the theory is formulated for the total virtual power, and there is no a priori partition into internal and external forces.

Poisson [1829, p. 400], before Piola had published his variational theories, criticized the methods of Lagrange for not being suitable for continuum mechanics. Piola was thereby stimulated to defend the variational approach to continuum mechanics; see the introduction of [Piola 1848] $]^{5}$. More than a hundred years later, Truesdell came up with a similar criticism in [Truesdell and Toupin 1960, §231] and charged the theory of Hellinger [1914, §3a] to fail through petitio principii, because the stress components would unmotivatedly appear in the constituting variational principle. To avoid this a priori notion of internal virtual work, Del Piero [2009] proposed an approach based on the objectivity criterion of Noll [1974]. Instead of the work, the virtual work of the external forces is required to be objective in his contribution. Hence, the balance laws can be deduced and used to prove Cauchy's stress theorem. Using in the virtual work of the external forces of an arbitrary subbody the relation between the surface force and the normal vector of the cutting surface, an expression of the internal virtual work is obtained. Knowing the form of the internal virtual work, the principle of virtual work can then be formulated a posteriori as being the balance between the virtual work of external and internal

${ }^{5}$ An English translation is given in [Piola 2014, Chapter 1, p. 4]. 
forces. Continuum mechanics based on requiring objectivity of the external virtual work (or power) can also be found in the textbook of Mariano and Galano [2015]. A similar derivation of the internal virtual work contribution is given in the earlier work of Murnaghan [1937].

In the nonvariational approach the stress principle of Euler and Cauchy defines the notion of stress in the sense that it restricts the interaction between subbodies to take place by contact surface forces only. Hence, the contact interaction between the subbodies is chosen as a basic concept. Together with the equilibrium of forces, the existence of the stress tensor follows from Cauchy's theorem. In contrast, in the variational theory as proposed by Germain [1972; 1973a; 1973b], the stress tensor field is defined as the quantity dual to the gradient of the virtual displacement field. By the application of the divergence theorem, the interaction mechanism between neighboring subbodies, i.e., the contact interaction, follows as a consequence. The latter is in the variational theory a derived concept but not an independent one. As mentioned above, the variational formulation is often criticized due to its a priori definition of the internal virtual work. Consequently, the question arises whether the internal virtual work contribution can be deduced from already known mechanical concepts, but still completely within the variational framework. This is meant to show the existence of the stress tensor, but without using the stress principle of Euler and Cauchy. It seems to the authors that such a derivation has already been obtained in [Piola 1848, Capitolo VI], ${ }^{6}$ "On the motion of a generic deformable body following the ideas of the modern scientists about the molecular actions", where Piola presented a nonlocal continuum theory deduced by the identification of the virtual work contributions of a discrete micromodel with the corresponding virtual work of a continuous macromodel, i.e., the continuum. As proposed by [dell'Isola et al. 2015a], we call this approach Piola's micro-macro identification procedure. Piola [1848, Capitolo II] $]^{7}$ used this micro-macro identification also for external forces when discussing line, surface, and volume forces. Having the discrete molecular and atomistic structure of a body in mind, Piola associated the placements of the material particles of a discrete system with the continuous placement field of the continuum evaluated at distinct points. With a clever scaling of the appearing force quantities, and in the limit of vanishing distances between the infinitely many material points and their neighbors, the sum of all virtual work contributions of the discrete material particles can be rewritten as an integral expression. It is this very integral expression which is identified with the virtual work contribution of the continuous macromodel. In his nonlocal continuum theory, Piola proposes a model in which each material point can interact with all the

\footnotetext{
${ }^{6}$ An English translation is given in [Piola 2014, Chapter 1, pp. 146-164] and commented on in [dell'Isola et al. 2015a].

${ }^{7}$ An English translation is given in [Piola 2014, Chapter 1, pp. 31-75].
} 
other material points of the body. The micro-macro identification procedure then leads straightforwardly to a virtual work contribution having the form of a sixfold integral over the body; see (10) in [dell'Isola et al. 2015a, p. 5]. A subsequent Taylor expansion of the variation of the quadratic distances between the material points, together with painful rearrangements of the force quantities, yields then the virtual work expressions of an $N$-th-gradient theory [dell'Isola et al. 2015a].

In this paper we aim to show that Piola's micro-macro identification procedure, which has fallen into oblivion until its translation into English by dell'Isola et al. [Piola 2014], is a suitable approach to derive the virtual work contributions for a continuum. More precisely, we want to address a derivation in which the internal virtual work contribution of the classical continuum is obtained directly from a micro-macro identification, which does not require the full Taylor expansion of Piola's nonlocal theory. Furthermore, we slightly modify Piola's line of arguments. Instead of motivating the appearing micromodel from the molecular structure of the continuum, we claim that such a micromodel is naturally included in the definition of an integral. We start from the concept of the volume integral appearing in the virtual work as being obtained by a dissection of the body into volume elements followed by a limit process with a refinement of these elements. In this way, the mathematical definition of the volume integral naturally provides a micro-macro relation between the infinitesimal volume elements and the continuum. The identification of the virtual work of the continuous macromodel with the virtual work of the discrete micromodel in the limit of vanishing volume elements can then be understood as Piola's micro-macro identification procedure. Within this context, we propose in addition a definition of stress as the internal force effects of the body on the micro level, which model the interaction between neighboring infinitesimal volume elements. The very same perception of stress has already been formulated by Boltzmann in his Populäre Schriften:

"What now concerns the forces which the volume elements of solid bodies mutually exert on each other, one must assume, that each volume element acts on only its direct neighbors, and that it exerts forces on all points adjacent to the cutting surface, which act just as if pulling threads under tension or pushing supporting bars were attached to it." 8

Within the limit process of vanishing volume elements, the summation of all virtual work contributions of the force interactions between the volume elements of the body leads to the appearance of the stress tensor in the internal virtual work contribution of the continuum. Hence, using the definition of the stress on the micro level of the infinitesimal volume elements in the sense of Boltzmann together with Piola's micro-macro identification procedure, the existence of the stress tensor and

${ }^{8}$ This is an English translation of [Boltzmann 1905, p. 297]. 
consequently the internal virtual work of the continuum can be derived without using the stress principle of Euler and Cauchy. The variational law of interaction, which requires the internal virtual work to vanish for all rigid virtual displacements, implies in a further step the symmetry of the stress tensor. With this alternative perspective on the concept of stress, it is possible to shed some light on the internal virtual work of the continuum and to formulate mechanics completely within a variational framework, in which the principle of virtual work and the variational law of interaction emerge as fundamental axioms of mechanics.

The outline of the paper is as follows. In Section 2, the required kinematics of the continuum is introduced. Section 3 proposes a variational theory of mechanics, mainly based on the insights of [Germain 1972; dell'Isola et al. 2012], with the principle of virtual work and the variational law of interaction as the basic axioms. The core of this paper is formulated in Section 4: based on an alternative definition of stress on the micro level of infinitesimal volume elements and Piola's micro-macro identification procedure, the virtual work contribution of the stress is derived on the macro level, together with the emerging stress tensor. In Section 5 the symmetry of the stress tensor is shown as a consequence of the variational law of interaction. The invariance of the virtual work with respect to different integral parametrizations and coordinate representations induces transformation properties of the appearing force quantities. A small selection of transformation properties for the stress is shown in Section 6. Section 7 derives the boundary value problem from the principle of virtual work for the classical assumption that external forces contribute either as volume forces in the interior of the body or as surface forces on the boundary. For the very same assumptions on the external forces, the integral balance laws are derived in Section 8. In the course of this, the inverse stress theorem appears which answers the question how a subbody interacts with its complement. Finally, conclusions are drawn in Section 9.

\section{Kinematics of the continuum}

In this section we introduce briefly the kinematical objects required for the variational formulation of continuum mechanics. The physical space is represented by the three-dimensional Euclidean vector space $\mathbb{E}^{3}$, equipped with an orthonormal basis $\left(\boldsymbol{e}_{x}^{I}, \boldsymbol{e}_{y}^{I}, \boldsymbol{e}_{z}^{I}\right)$ and an origin $O$, subsequently called the $I$-system. A point of the physical space is addressed by the position vector $x \in \mathbb{E}^{3}$. The cartesian coordinate representation of the position vector $\boldsymbol{x}$ in the $I$-system is denoted as

$$
{ }_{I} \boldsymbol{x}:=\left(\begin{array}{l}
x \\
y \\
z
\end{array}\right) \quad \text { with } \boldsymbol{x}=x \boldsymbol{e}_{x}^{I}+y \boldsymbol{e}_{y}^{I}+z \boldsymbol{e}_{z}^{I}
$$




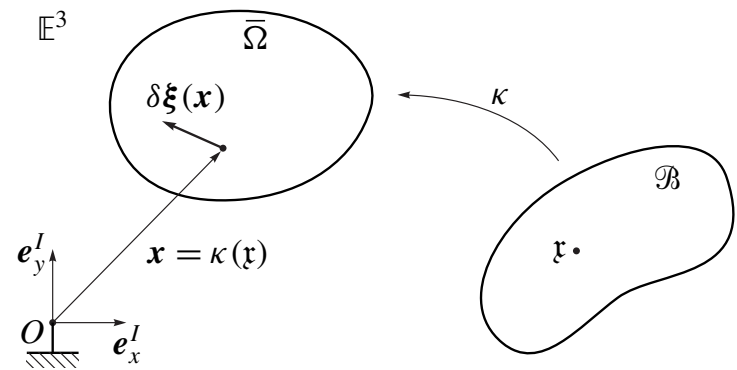

Figure 1. The placement $\kappa$ of the body $\mathscr{B}$ into the Euclidean vector space $\mathbb{E}^{3}$. At every material point $\boldsymbol{x}$ in the deformed configuration $\bar{\Omega}$, a virtual displacement $\delta \boldsymbol{\xi}(\boldsymbol{x})$ can be evaluated.

The body $\mathscr{\Re}$, as suggested by [Noll 1959; Segev 1986], is a three-dimensional compact differentiable manifold. A point of the body manifold $\mathfrak{x} \in \mathscr{B}$ is called a material point of $\mathscr{B}$. The placement of $\mathscr{B}$, as depicted in Figure 1 , is the embedding of the body into the physical space, i.e., the mapping $\kappa: \mathscr{B} \rightarrow \mathbb{E}^{3}$. Embeddings as proper injective immersions respect the principle of impenetrability and the permanence of matter; see [Truesdell and Toupin 1960, §16] for their specific definitions. The image $\bar{\Omega}=\kappa(\mathscr{B}) \subset \mathbb{E}^{3}$ is the region in the physical space occupied by the body manifold and is called the deformed configuration. The deformed configuration as a closed subset ${ }^{9}$ can be written as a disjoint union $\bar{\Omega}=\Omega \cup \partial \Omega$ of the interior $\Omega$ and the boundary $\partial \Omega$ of the deformed configuration, respectively. A spatial point $\boldsymbol{x} \in \bar{\Omega}$ is called a material point in the deformed configuration $\bar{\Omega}$.

A variational family of placements is a differentiable parametrization of placements $\bar{\kappa}(\varepsilon, \mathfrak{x})$ with respect to a single parameter $\varepsilon \in \mathbb{R}$, such that $\kappa(\mathfrak{x})=\bar{\kappa}\left(\varepsilon_{0}, \mathfrak{x}\right)$. The virtual displacement field $\delta \xi: \bar{\Omega} \rightarrow \mathbb{E}^{3}$ in the deformed configuration is the smooth vector field over $\bar{\Omega}$ defined as

$$
\delta \boldsymbol{\xi}(\boldsymbol{x}):=\frac{\partial \bar{\kappa}}{\partial \varepsilon}\left(\varepsilon_{0}, \kappa^{-1}(\boldsymbol{x})\right),
$$

which corresponds to the variation of the placement $\kappa$ evaluated at the material point $\mathfrak{x}=\kappa^{-1}(\boldsymbol{x})$. When no body manifold is available or not of interest as for instance in fluid mechanics where often only control volumes in space are considered, the virtual displacement field can be directly defined as a smooth vector field over the deformed configuration $\bar{\Omega}$. By multiplying (2) with a small $\delta \varepsilon=\left(\varepsilon-\varepsilon_{0}\right)$, the resulting fields can be interpreted as infinitesimal displacements of the deformed

\footnotetext{
${ }^{9} \mathrm{~A}$ compact manifold embedded into the Euclidean space $\mathbb{E}^{3}$ is a closed subset of $\mathbb{E}^{3}$, as is evident by the following two propositions from set theory [Munkres 2000]. Proposition 1: The image of a compact set under a continuous map is compact. Proposition 2: A compact set of a Hausdorff space is closed. Since an embedding is a continuous map and the Euclidean vector space is a Hausdorff space, the assertion follows immediately.
} 
configuration. A subset of such displacements are displacements which shift the deformed configuration infinitesimally as if it was the configuration of a rigid body. The corresponding virtual displacements constitute the subset of rigid virtual displacements $\delta \xi_{\text {rig }}$ which can be parametrized for instance as

$$
\delta \boldsymbol{\xi}_{\text {rig }}(\boldsymbol{x})=\delta \boldsymbol{r}_{O}+\delta \boldsymbol{\phi} \times \boldsymbol{x},
$$

where $\delta \boldsymbol{r}_{O} \in \mathbb{E}^{3}$ and $\delta \boldsymbol{\phi} \in \mathbb{E}^{3}$ denote the virtual displacement of the origin $O$ and the virtual rotation, respectively. The virtual rotation vector $\delta \phi$ or, more precisely, the virtual rotation operator $\delta \phi \times$ will mainly be used throughout the text instead of the skew-symmetric linear map $\tilde{\boldsymbol{\phi}}(\varepsilon)$ with $\tilde{\boldsymbol{\phi}}\left(\varepsilon_{0}\right)=0$, which provides via $\overline{\boldsymbol{\xi}}(\varepsilon, \boldsymbol{x})=$ $\exp (\tilde{\boldsymbol{\phi}}(\varepsilon)) \boldsymbol{x}$ a variational family of rigid rotations.

\section{The axioms of mechanics}

Once we have introduced the kinematics of the continuum, we briefly reconsider the axiomatic scheme of variational continuum mechanics proposed by Germain [1972; 1973b]. Following Lagrange's ideas, we start with the mechanical definition of forces and consider them as quantities dual to the virtual displacements. As a consequence and as stated for instance in [dell'Isola et al. 2015b; 2011; 2012], we therefore accept the idea of considering forces as distributions in the sense of L. Schwartz [1951]. This definition already uses the concept of duality and motivates the formulation of the equilibrium conditions in variational form, which is the first axiom, the principle of virtual work. In an upcoming step, internal and external forces are defined in order to impose a certain variational condition on the internal forces. This is the second axiom, the variational law of interaction.

Definition 1 (mechanical definition of force). We define force as a linear functional $\boldsymbol{F}$ on the space of virtual displacement fields $\delta \boldsymbol{\xi}$, which associates with every $\delta \boldsymbol{\xi}$ a real number $\delta W=\boldsymbol{F}(\delta \xi)$ called virtual work.

For a continuum, the space of virtual displacement fields is given by all smooth vector fields over the body $\bar{\Omega}$. Choosing appropriate compact supports for these vector fields, and following the results of the theory of distributions, forces can be represented by the map

$$
\delta \boldsymbol{\xi} \mapsto \delta W=\boldsymbol{F}(\delta \boldsymbol{\xi}) \quad \text { with } \boldsymbol{F}(\delta \boldsymbol{\xi})=\sum_{k=0}^{N} \int_{\bar{\Omega}} \frac{\partial^{k}}{\partial \boldsymbol{x}^{k}}(\delta \boldsymbol{\xi}) \mid \mathrm{d} \boldsymbol{F}^{k},
$$

where $\partial^{k} / \partial \boldsymbol{x}^{k}$ denotes the $k$-th partial derivative with respect to $\boldsymbol{x}$, the force measure $\mathrm{d} \boldsymbol{F}^{k}$ is a tensor-valued measure of rank $k+1$ having support in $\bar{\Omega}$, and the symbol $\mid$ stands for the complete contraction of the tensors, i.e., a $(k+1)$ contraction. We refer to [dell'Isola et al. 2015b] for more technical details about 
this result. Remark that (4) gives a much larger spectrum of force representations than required for the classical continuum theory. However, it is precisely one of the aims of this paper to deduce, by an appropriate micro-macro identification, the force representations for the interactions of the classical theory.

Contained in the integral of (4) is that the $k$-th derivative of the virtual displacement and the corresponding force measure $\mathrm{d} \boldsymbol{F}^{k}$ have to be evaluated at the very same point $\boldsymbol{x} \in \bar{\Omega}$ and are then to be contracted, which constitutes the duality pairing and which may be phrased as follows.

Forces, as abstract entities, are perceived by imposing test displacements (and their derivatives) at every point $\boldsymbol{x} \in \bar{\Omega}$, in order to characterize them completely in magnitude and direction.

Note that the magnitude does not require the concept of a metric in this context. The magnitude is given by the virtual work (4), which is per definition a metricindependent scalar. This scalar furthermore does not depend on the chosen coordinate representation of $\delta \boldsymbol{\xi}$ and $\mathrm{d} \boldsymbol{F}^{k}$, nor on the parametrization of the appearing integral. This motivates regarding the virtual work as the invariant quantity in mechanics. Conversely, the invariance of the virtual work provides all the transformation rules for the occurring force quantities when a coordinate representation is chosen, or when the parametrization of the integral is changed. The physical units of forces, virtual displacements, and the virtual work are $[\boldsymbol{F}]=\mathrm{N},[\delta \boldsymbol{\xi}]=\mathrm{m}$, and $[\delta W]=\mathrm{Nm}=\mathrm{J}$, respectively. Based on the definition of force, we may now state the first axiom, which is the equilibrium conditions of a body in variational form.

Axiom 1 (principle of virtual work). Let the force measures $\mathrm{d} \boldsymbol{F}^{k}$ contain the totality of forces acting on the body in its deformed configuration $\bar{\Omega}$. For the body to be in equilibrium, the overall virtual work $\delta W_{\text {tot }}$ generated by $\mathrm{d} \boldsymbol{F}^{k}$ has to vanish for all virtual displacements $\delta \xi$ :

$$
\delta W_{\mathrm{tot}}=\sum_{k=0}^{N} \int_{\bar{\Omega}} \frac{\partial^{k}}{\partial \boldsymbol{x}^{k}}(\delta \boldsymbol{\xi}) \mid \mathrm{d} \boldsymbol{F}^{k}=0 \quad \text { for all } \delta \boldsymbol{\xi} .
$$

The principle of virtual work (5) requires the totality of forces acting on the body, which encompasses both the internal and the external forces of $\bar{\Omega}$, as they are defined below. This totality represented by the force measures $\mathrm{d} \boldsymbol{F}^{k}(\boldsymbol{x})$ is obtained by the pointwise summation of the individual force contributions $\mathrm{d} \boldsymbol{F}_{i}^{k}(\boldsymbol{x})$, since forces as linear functionals are additive. Furthermore, (5) has to hold for arbitrary virtual displacement fields $\delta \xi$. This arbitrariness implies that the virtual work also has to vanish for all suitably regular subbodies.

Definition 2 (internal and external forces). Let $K$ be a subsystem of $\bar{\Omega}$, that is, $K \subseteq \bar{\Omega}$. We call $\boldsymbol{F}^{\mathrm{i}}$ an internal force of $K$ if it is exerted from material points 
$\boldsymbol{x} \in K$ on other material points $\boldsymbol{y} \in K$. We call $\boldsymbol{F}^{\mathrm{e}}$ an external force of $K$ if it is exerted from the environment of $K$ on some material points $\boldsymbol{x} \in K$.

Internal forces describe the force interactions among the points $x \in K$ exclusively, whereas external forces act from outside on the points $\boldsymbol{x} \in K$. The classification into internal and external forces therefore depends strongly on the considered subsystem. An external force $\boldsymbol{F}$ of $K \subset \bar{\Omega}$, which acts from a subset $B \subset \bar{\Omega} \backslash K$ on some material points in $K$, is an internal force of any subsystem $C \cup K$ with $C \supseteq B$. This definition is very classical and strongly related to mechanical modeling. The same classification can be found for instance in [Hellinger 1914, pp. 637-638] ${ }^{10}$ or [Germain 1973b] ${ }^{11}$. One must bear in mind that every mechanical interaction which one desires to model, in order to predict an observation, has to be introduced explicitly in the mathematical framework of a mechanical theory. Based on the definition of the internal forces, we may now state the second axiom.

Axiom 2 (variational law of interaction ${ }^{12}$ ). Let $\delta W^{\mathrm{i}}$ denote the virtual work generated by the totality of internal forces measures $\mathrm{d} \boldsymbol{F}^{\mathrm{i}, k}$ of an arbitrary subsystem $K \subseteq \bar{\Omega}$. It then holds that $\delta W^{\mathrm{i}}$ vanishes under each rigid virtual displacement field $\delta \xi_{\text {rig }}$ as specified in (3):

$$
\delta W^{\mathrm{i}}=\sum_{k=0}^{N} \int_{K} \frac{\partial^{k}}{\partial \boldsymbol{x}^{k}}(\delta \boldsymbol{\xi}) \mid \mathrm{d} \boldsymbol{F}^{\mathrm{i}, k}=0 \quad \text { for all } \delta \boldsymbol{\xi}_{\mathrm{rig}} .
$$

From a mechanical point of view, the variational law of interaction can be interpreted as follows: the totality of internal force measures $\mathrm{d} \boldsymbol{F}^{\mathrm{i}, k}$ of the subsystem $K \subseteq \bar{\Omega}$ is not perceived from the outside when the subsystem as a whole is virtually moved like a rigid body, or in other words, one does not have to work against the total internal force under any virtual rigid body motion. It has to be mentioned that (6) does not give any conditions on the force measures $\mathrm{d} \boldsymbol{F}^{\mathrm{i}, k}$ of order $k>1$.

${ }^{10}$ Also see the translation [Eugster and dell'Isola 2017b, p. 11]: "Thereby primarily the [following] difference must be clarified, if the force effects are external, i.e., [the effects] have their cause in the relation to media and sources of effects located outside the considered medium (long-range forces, pressures at the boundary and such like), or internal, i.e., [the effects] are based on the material constitution of the particular medium and the mutual effects of the particles thereof."

11 "The various "forces" which act on the mechanical system are divided in a very classical way into two classes: external forces which represent the dynamical effects on $S$ due to the interaction with other systems which have no common part with $S$, and internal forces which represent the mutual dynamical effects of subsystems of $S$; for instance, if $S_{i}$ and $S_{j}$ are two disjoint subsystems of $S$, the action of $S_{i}$ on $S_{j}$ represents "external forces" acting on $S_{j}$, but "internal forces" if one considers the system $S$ itself."

${ }^{12}$ Denoted by Germain [1973b] as axiom of power of internal forces. We prefer to call it variational law of interaction as it is the variational version of the law of interaction proposed by Glocker [2001, Chapter 2]. 
In contrast to the first axiom, the variational law of interaction requires a metric to extract from it the rigid virtual displacement fields $\delta \xi_{\text {rig }}$, for which the variations are evaluated. By using their specific parametrization (3), the universal quantifier in (6) implies that the variations have to be carried out independently for the two kinematic quantities $\delta \boldsymbol{r}_{O}$ and $\delta \boldsymbol{\phi}$. This leads to the resultant forces as the quantities dual to $\delta \boldsymbol{r}_{O}$, but also to the first occurrence of (resultant) moments as the quantities dual to $\delta \phi$. The fact that precisely three equations are obtained for the resultant forces, and another three for the moments, is attributed to the specific form (3) of the rigid virtual displacement field in $\mathbb{E}^{3}$ and would be different if another metric space would have been chosen for the embedding. Note that the moments always lead back to the variational law of interaction (6) in our approach, as no metric is needed in the principle of virtual work (5), nor in the representation of forces (4).

\section{From Piola's micro-macro identification to the virtual work of stress}

Equation (4) shows us the wide variety of possible force interactions in a continuum. In most variational formulations of continuum mechanics, as, e.g., [Hellinger 1914; Germain 1972; 1973a; 1986; Salençon 2000; Salençon 2001], only distinct subsets of these force representations are postulated for the internal and external virtual work. In this section, we show how these virtual work contributions of the classical continuum can be derived using Piola's micro-macro identification procedure.

Integration always consists of a dissection of a region into simple elements, followed by a limit process with a refinement of these elements. We sketch this process for the Riemann integral over the body volume with a dissection of the deformed configuration $\Omega$ into cuboids and an approximation from the inside. As a sort of a lower Darboux sum, an approximation from the inside sums the contributions of the inner cuboids, i.e., the cuboids lying completely within the domain $\bar{\Omega}$; see Figure 2. In what follows, we will denote the position vector of the center of a cuboid $i j k$ with respect to the $I$-system by ${ }_{I} \boldsymbol{x}_{i j k}=\left(x_{i}, y_{j}, z_{k}\right)^{\mathrm{T}}$. The indices $i, j, k$ appearing in the subsequent sums range always such that all inner cuboids are taken into account. Without loss of generality, we assume all the infinitesimal volume elements to be of the same size $\Delta V=\Delta x \Delta y \Delta z$, where $\Delta x, \Delta y, \Delta z$ denote the lengths of the corresponding edges. Accordingly, the volume integral over the body $\Omega$ of a function $w(\boldsymbol{x})$ is obtained when the limit

$$
\int_{\Omega} w(\boldsymbol{x}) \mathrm{d} v=\lim _{\Delta V \rightarrow 0} \sum_{i, j, k} w\left(x_{i}, y_{j}, z_{k}\right) \Delta V
$$

does exist.

It is now this type of volume integral which we use in the context to interpret Piola's micro-macro identification procedure. Crucial for the following is that the 


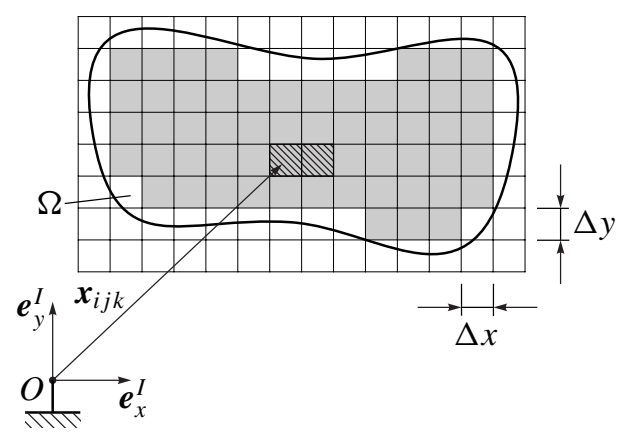

Figure 2. Dissection of the deformed configuration $\Omega$ into cuboids and approximation of the Riemann integral from the inside. The inner cuboids lying completely within the domain $\bar{\Omega}$ are colored in gray.

discrete approximation of the body by finite volume elements can already be considered as a mechanical model, i.e., the micromodel. The identification procedure is then performed as follows.

- The virtual work contributions $\Delta W$ are stated for the force interactions that occur (or are modeled) in the discrete micromodel.

- The virtual displacement of a discrete volume element $i j k$ is identified with the continuous virtual displacement field evaluated at an arbitrary point inside the volume element - for instance $\delta \boldsymbol{\xi}\left(\boldsymbol{x}_{i j k}\right)$ when it is evaluated at the center point $\boldsymbol{x}_{i j k}$.

- In the limit of vanishing volume elements $\Delta V \rightarrow 0$, the virtual work contribution of the continuous macromodel $\delta W$ is identified with the virtual work of the discrete micromodel $\Delta W$, i.e., $\delta W=\lim _{\Delta V \rightarrow 0} \Delta W$.

Obviously, the same procedure can also be applied for lower dimensional integrations, to obtain force interactions on lines or surfaces.

What now follows for the external forces can be found similarly in [Piola 1848, $\S \S 31-32]^{13}$, despite the fact that Piola uses a reference configuration to address the material points. One kind of force interaction that we allow to be exerted from the environment on the body, i.e., as an external force, leads to the virtual work contribution

$$
\Delta W_{v}^{\mathrm{e}}=\sum_{i, j, k} \delta \xi\left(\boldsymbol{x}_{i j k}\right) \cdot \Delta \boldsymbol{F}_{v}\left(\boldsymbol{x}_{i j k}\right)=\sum_{i, j, k} \delta \boldsymbol{\xi}\left(\boldsymbol{x}_{i j k}\right) \cdot \frac{\Delta \boldsymbol{F}_{v}\left(\boldsymbol{x}_{i j k}\right)}{\Delta V} \Delta V
$$

${ }^{13}$ An English translation is given in [Piola 2014, Chapter 1, pp. 51-55]. 
of the discrete micromodel. Remark that the virtual work of a single cuboid is obtained by the duality pairing between the virtual displacement $\delta \boldsymbol{\xi}\left(\boldsymbol{x}_{i j k}\right)$ and the force $\Delta \boldsymbol{F}_{v}\left(\boldsymbol{x}_{i j k}\right)$, which can be considered as a force in the sense of Newton. The virtual work $\delta W_{v}^{\mathrm{e}}$ of the external force effect appearing in the continuous macromodel is then identified with the micromodel by

$$
\delta W_{v}^{\mathrm{e}}=\lim _{\Delta V \rightarrow 0} \Delta W_{v}^{\mathrm{e}}=\int_{\Omega} \delta \xi \cdot \boldsymbol{f}_{v} \mathrm{~d} v,
$$

where the existence of the limit

$$
\boldsymbol{f}_{v}(\boldsymbol{x}):=\lim _{\Delta V \rightarrow 0} \frac{\Delta \boldsymbol{F}_{v}\left(\boldsymbol{x}_{i j k}\right)}{\Delta V}
$$

has to be assumed. Equation (10) is nothing else but the definition of a volume force with unit $\left[f_{v}\right]=\mathrm{N} / \mathrm{m}^{3}$.

The forces exerted by the environment on the body via the surface of the deformed configuration $\partial \Omega$ can be modeled in a similar way. This leads then to a surface force $f_{a}$ with unit $\left[f_{a}\right]=\mathrm{N} / \mathrm{m}^{2}$ and to the corresponding virtual work contribution $\delta \boldsymbol{\xi} \cdot \boldsymbol{f}_{a}$, such that the virtual work of external forces for the continuum is of the form

$$
\delta W^{\mathrm{e}}=\int_{\Omega} \delta \boldsymbol{\xi} \cdot \boldsymbol{f}_{v} \mathrm{~d} v+\int_{\partial \Omega} \delta \boldsymbol{\xi} \cdot \boldsymbol{f}_{a} \mathrm{~d} a
$$

We could also introduce further virtual work contributions of external force effects that are acting on surfaces, lines, or even points inside the body.

On the micro level of volume elements, one may also introduce force effects between the individual volume elements. This is exactly the idea, which enables us to give a verbal definition of stress on the discrete micro level of infinitesimal volume elements in the sense of Boltzmann.

Definition 3 (stress). We define stress as the internal force effects of the body that model the mutual force interactions between neighboring infinitesimal volume elements sharing the same surfaces.

We want to mention that this definition explicitly excludes the interaction between cuboids sharing the same edges and wedge points. The upcoming derivation shows how the three stress vectors of the continuous macro level emerge in the virtual work from Definition 3 together with Piola's micro-macro identification procedure.

In a first step, we consider two neighboring cuboids with coordinates ${ }_{I} \boldsymbol{x}_{i j k}=$ $\left(x_{i}, y_{j}, z_{k}\right)^{\mathrm{T}}$ and ${ }_{I} \boldsymbol{x}_{(i+1) j k}=\left(x_{i+1}, y_{j}, z_{k}\right)^{\mathrm{T}}$, which are aligned in the $\boldsymbol{e}_{x}^{I}$-direction as depicted in Figure 2 as hatched elements. As all volume elements are of the same size, it holds that $x_{i+1}=x_{i}+\Delta x$. Figure 3 shows a close-up of these two cuboids together with their virtual displacements $\delta \xi$ and their force interactions 


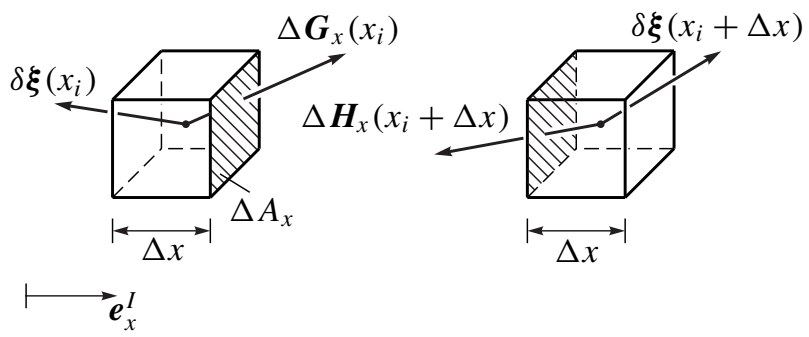

Figure 3. Force interaction between two neighboring volume elements aligned in the $\boldsymbol{e}_{x}^{I}$-direction.

$\Delta \boldsymbol{G}_{x}$ and $\Delta \boldsymbol{H}_{x}$. For the sake of brevity, we omit in the figures as well as in the upcoming formulas the dependence of $\delta \boldsymbol{\xi}, \Delta \boldsymbol{G}_{x}$, and $\Delta \boldsymbol{H}_{x}$ on the coordinates $y_{j}$ and $z_{k}$. The discrete virtual work contribution of the two adjacent cuboids by the force increments $\Delta \boldsymbol{G}_{x}$ and $\Delta \boldsymbol{H}_{x}$ is

$$
\Delta W^{\mathrm{s}}=\delta \boldsymbol{\xi}\left(x_{i}\right) \cdot \Delta \boldsymbol{G}_{x}\left(x_{i}\right)+\delta \boldsymbol{\xi}\left(x_{i}+\Delta x\right) \cdot \Delta \boldsymbol{H}_{x}\left(x_{i}+\Delta x\right)
$$

with $\Delta \boldsymbol{G}_{x}$ being the force with unit $\left[\Delta \boldsymbol{G}_{x}\right]=\mathrm{N}$ exerted from the right cuboid on the left cuboid and $\Delta \boldsymbol{H}_{x}$ being the force with unit $\left[\Delta \boldsymbol{H}_{x}\right]=\mathrm{N}$ exerted from the left cuboid on the right cuboid. To relate the forces $\Delta \boldsymbol{G}_{x}$ and $\Delta \boldsymbol{H}_{x}$ in (12) to each other, we introduce forces $\Delta \boldsymbol{F}_{x}$ and $\Delta \boldsymbol{C}_{x}$ such that

$$
\Delta \boldsymbol{G}_{x}\left(x_{i}\right)=\Delta \boldsymbol{F}_{x}\left(x_{i}\right)+\Delta \boldsymbol{C}_{x}\left(x_{i}\right) \text { and } \Delta \boldsymbol{H}_{x}\left(x_{i}+\Delta x\right)=-\Delta \boldsymbol{F}_{x}\left(x_{i}\right) .
$$

Note that we intentionally violate here the principle of action and reaction by the force $\Delta \boldsymbol{C}_{x}$ : the principle of action and reaction does not constitute an independent axiom in our approach, but is contained in the variational law of interaction. Consequently, we do not apply action equals reaction by setting $\Delta \boldsymbol{C}_{x}=0$, but leave it to the variational law of interaction on the macro level to decide later whether the force $\Delta \boldsymbol{C}_{x}$ is needed in (13). With the help of (13), the virtual work contribution (12) of the two adjacent cuboids becomes

$$
\begin{aligned}
\Delta W^{\mathrm{s}} & =\delta \boldsymbol{\xi}\left(x_{i}\right) \cdot \Delta \boldsymbol{C}_{x}\left(x_{i}\right)+\delta \boldsymbol{\xi}\left(x_{i}\right) \cdot \Delta \boldsymbol{F}_{x}\left(x_{i}\right)-\delta \boldsymbol{\xi}\left(x_{i}+\Delta x\right) \cdot \Delta \boldsymbol{F}_{x}\left(x_{i}\right) \\
& =\delta \boldsymbol{\xi}\left(x_{i}\right) \cdot \Delta \boldsymbol{C}_{x}\left(x_{i}\right)-\left(\delta \boldsymbol{\xi}\left(x_{i}+\Delta x\right)-\delta \boldsymbol{\xi}\left(x_{i}\right)\right) \cdot \Delta \boldsymbol{F}_{x}\left(x_{i}\right) \\
& =\delta \boldsymbol{\xi}\left(x_{i}\right) \cdot \Delta \boldsymbol{C}_{x}\left(x_{i}\right)-\left(\delta \boldsymbol{\xi}\left(x_{i}+\Delta x\right)-\delta \boldsymbol{\xi}\left(x_{i}\right)\right) \frac{1}{\Delta x} \cdot \Delta x \Delta \boldsymbol{F}_{x}\left(x_{i}\right) .
\end{aligned}
$$

We denote by $\Delta A_{x}:=\Delta y \Delta z$ the surface element that is shared by the adjacent cuboids and rewrite (14) as

$$
\Delta W^{\mathrm{s}}=\left(\delta \boldsymbol{\xi}\left(x_{i}\right) \cdot \frac{\Delta \boldsymbol{C}_{x}\left(x_{i}\right)}{\Delta V}-\frac{\delta \boldsymbol{\xi}\left(x_{i}+\Delta x\right)-\delta \boldsymbol{\xi}\left(x_{i}\right)}{\Delta x} \cdot \frac{\Delta \boldsymbol{F}_{x}\left(x_{i}\right)}{\Delta A_{x}}\right) \Delta V .
$$



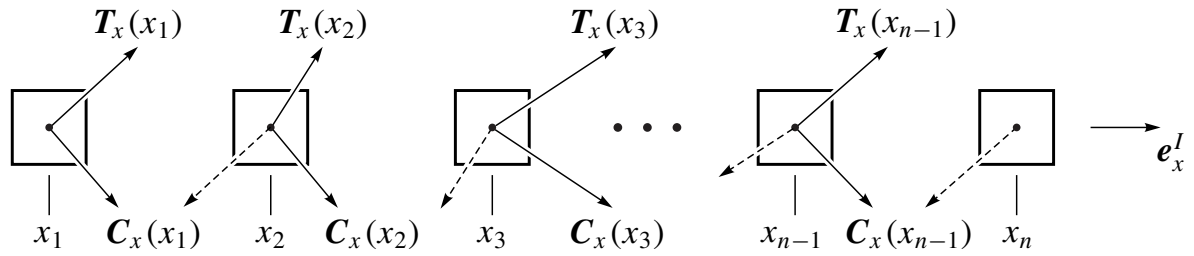

Figure 4. Mutual force interactions between the members of the row of inner cuboids in the direction $\boldsymbol{e}_{x}^{I}$ at the position $y_{j}, z_{k}$.

Furthermore, we introduce in (15) the abbreviations

$$
\begin{aligned}
\boldsymbol{C}_{x}\left(x_{i}\right) & :=\frac{\Delta \boldsymbol{C}_{x}\left(x_{i}\right)}{\Delta V}, \\
\boldsymbol{T}_{x}\left(x_{i}\right) & :=\frac{\Delta \boldsymbol{F}_{x}\left(x_{i}\right)}{\Delta A_{x}}, \\
D_{x} \delta \boldsymbol{\xi}\left(x_{i}\right) & :=\frac{\delta \boldsymbol{\xi}\left(x_{i}+\Delta x\right)-\delta \boldsymbol{\xi}\left(x_{i}\right)}{\Delta x},
\end{aligned}
$$

where $\boldsymbol{C}_{x}$ is the incremental volume force with unit $\left[\boldsymbol{C}_{x}\right]=\mathrm{N} / \mathrm{m}^{3}, \boldsymbol{T}_{x}$ is the incremental stress vector with unit $\left[\boldsymbol{T}_{x}\right]=\mathrm{N} / \mathrm{m}^{2}$, and $D_{x} \delta \boldsymbol{\xi}$ is the dimensionless differential quotient. Inserting the abbreviations (16) in (15), we obtain

$$
\Delta W^{\mathrm{s}}=\delta \boldsymbol{\xi}\left(x_{i}\right) \cdot \boldsymbol{C}_{x}\left(x_{i}\right) \Delta V-D_{x} \delta \boldsymbol{\xi}\left(x_{i}\right) \cdot \boldsymbol{T}_{x}\left(x_{i}\right) \Delta V .
$$

Equation (17) is the virtual work contribution of the mutual force interaction of two neighboring cuboids, which is the interaction of the two cuboids by internal short-range forces. As such, $\Delta W^{\mathrm{s}}$ will contribute in the limit to the internal virtual work $\delta W^{\mathrm{i}}$ in (6).

Figure 4 depicts the row of inner cuboids aligned in the $\boldsymbol{e}_{x}^{I}$-direction at the position $y_{j}, z_{k}$, together with all mutual force interactions that occur between each pair of neighboring cuboids. By adding all these force interactions to $\Delta W^{\mathrm{s}}$ in (17), we get the virtual work contribution of this entire row as

$$
\begin{aligned}
\Delta W^{\mathrm{s}}=\sum_{i=1}^{n-1} \delta \boldsymbol{\xi}\left(x_{i}, y_{j}, z_{k}\right) \cdot \boldsymbol{C}_{x}\left(x_{i}, y_{j}, z_{k}\right) \Delta V \\
\\
\quad-D_{x} \delta \boldsymbol{\xi}\left(x_{i}, y_{j}, z_{k}\right) \cdot \boldsymbol{T}_{x}\left(x_{i}, y_{j}, z_{k}\right) \Delta V .
\end{aligned}
$$

Summation of all the cuboid rows in the $\boldsymbol{e}_{x}^{I}$-direction leads to

$$
\Delta W^{\mathrm{s}}=\sum_{i, j, k}\left(\delta \boldsymbol{\xi} \cdot \boldsymbol{C}_{x}\right)_{i j k} \Delta V-\left(D_{x} \delta \boldsymbol{\xi} \cdot \boldsymbol{T}_{x}\right)_{i j k} \Delta V,
$$

which corresponds to a summation over all inner cuboids of the deformed configuration $\Omega$. The force interactions $\left(\boldsymbol{C}_{y}, \boldsymbol{T}_{y}\right)$ and $\left(\boldsymbol{C}_{z}, \boldsymbol{T}_{z}\right)$ to the neighboring cuboids 
in the $y$-and $z$-directions contribute in a similar way. Consequently, the entire virtual work of stress of the discrete micromodel is

$$
\begin{aligned}
\Delta W^{\mathrm{S}}=\sum_{i, j, k}\left(\delta \boldsymbol{\xi} \cdot \boldsymbol{C}_{x}+\delta \boldsymbol{\xi} \cdot \boldsymbol{C}_{y}+\delta \boldsymbol{\xi} \cdot \boldsymbol{C}_{z}\right)_{i j k} \Delta V \\
-\left(D_{x} \delta \boldsymbol{\xi} \cdot \boldsymbol{T}_{x}+D_{y} \delta \boldsymbol{\xi} \cdot \boldsymbol{T}_{y}+D_{z} \delta \boldsymbol{\xi} \cdot \boldsymbol{T}_{z}\right)_{i j k} \Delta V,
\end{aligned}
$$

which models all mutual force interactions between the neighboring inner cuboids.

The virtual work $\delta W^{\mathrm{s}}$ of the mutual force interactions on the macro level is then identified with the virtual work of the micromodel (20) by taking the limit

$$
\delta W^{\mathrm{s}}=\lim _{\Delta V \rightarrow 0} \Delta W^{\mathrm{s}},
$$

which requires the limits of the objects in (16) to exist. We denote them by

$$
\begin{aligned}
\boldsymbol{c}_{x}\left({ }_{I} \boldsymbol{x}\right) & :=\lim _{\Delta V \rightarrow 0} \boldsymbol{C}_{x}\left(x_{i}, y_{j}, z_{k}\right), \\
\boldsymbol{t}_{x}\left({ }_{I} \boldsymbol{x}\right) & :=\lim _{\Delta A_{x} \rightarrow 0} \boldsymbol{T}_{x}\left(x_{i}, y_{j}, z_{k}\right), \\
\delta \boldsymbol{\xi}_{, x}\left({ }_{I} \boldsymbol{x}\right) & :=\lim _{\Delta x \rightarrow 0} D_{x} \delta \boldsymbol{\xi}\left(x_{i}, y_{j}, z_{k}\right) .
\end{aligned}
$$

Similar to (16), $\boldsymbol{c}_{x}$ is a volume force with unit $\left[\boldsymbol{c}_{x}\right]=\mathrm{N} / \mathrm{m}^{3}, \boldsymbol{t}_{x}$ is the stress vector with unit $\left[\boldsymbol{t}_{x}\right]=\mathrm{N} / \mathrm{m}^{2}$, and $\delta \boldsymbol{\xi}_{, x}$ is the dimensionless partial derivative of $\delta \boldsymbol{\xi}$ with respect to $x$. The corresponding contributions in the $y$ - and $z$-directions are denoted accordingly. Using (20) and (22) and defining

$$
\boldsymbol{c}:=\boldsymbol{c}_{x}+\boldsymbol{c}_{y}+\boldsymbol{c}_{z},
$$

we can identify the virtual work of the continuous macromodel as

$$
\delta W^{\mathrm{s}}=\int_{\Omega}\left(\delta \boldsymbol{\xi} \cdot \boldsymbol{c}-\delta \boldsymbol{\xi}_{, x} \cdot \boldsymbol{t}_{x}-\delta \boldsymbol{\xi}_{, y} \cdot \boldsymbol{t}_{y}-\delta \boldsymbol{\xi}_{, z} \cdot \boldsymbol{t}_{z}\right) \mathrm{d} v,
$$

where $\mathrm{d} v$ denotes the volume element in the deformed configuration. In addition to the term $\delta \xi \cdot c$, the stress vectors $\boldsymbol{t}_{i}$ of the three orthogonal spatial directions $i=x, y, z$ contribute to $\delta W^{\mathrm{s}}$, paired with the corresponding partial derivatives $\delta \boldsymbol{\xi}_{, i}$.

The reason to take the limits like in (22) and not in any other form is motivated as follows. If we assume $\Delta \boldsymbol{C}_{x}=0$, then $\Delta \boldsymbol{G}_{x}$ and $\Delta \boldsymbol{H}_{x}$ in (13) can be interpreted as two mutual force distributions, equal in size and opposite in direction, which are infinitesimally shifted against each other. If we would factor out $\Delta V$ and already take the limit $\Delta V \rightarrow 0$ in the second line of (14), the volume force $\Delta \boldsymbol{F}_{x} / \Delta V$, paired with the vanishing difference $\delta \xi\left(x_{i}+\Delta x\right)-\delta \xi\left(x_{i}\right)$, would have to be infinitely large to produce a nonvanishing contribution to the integral (" $0 \cdot \infty$ "). The mere difference $\delta \xi\left(x_{i}+\Delta x\right)-\delta \boldsymbol{\xi}\left(x_{i}\right)$ alone is not strong enough to keep bounded volume forces for $\Delta V \rightarrow 0$ in the integral. However, by using the differential quotient $(1 / \Delta x)\left[\delta \xi\left(x_{i}+\Delta x\right)-\delta \xi\left(x_{i}\right)\right]$ as in the third line of (14), the aforementioned 
difference is strengthened by the division with $\Delta x$ and a nonzero expression is obtained. At the same time, the volume force $\Delta \boldsymbol{F}_{x} / \Delta V$, which tends to infinity for $\Delta V \rightarrow 0$, has to be moderated to a surface force $\Delta \boldsymbol{F}_{x} / \Delta A_{x}$, which, after the limit process, is called the stress vector $\boldsymbol{t}_{x}$.

The choice of a particular cartesian parametrization of the deformed configuration $\Omega$ in the formulation (24) was inevitable. In order to get rid of this particular choice, we bring (24) into a coordinate-free form, in which the stress tensor emerges as a Euclidean second-order tensor. Using the cartesian coordinates $(x, y, z)$ with the orthonormal basis vectors $\left(\boldsymbol{e}_{x}^{I}, \boldsymbol{e}_{y}^{I}, \boldsymbol{e}_{z}^{I}\right)$, the partial derivative with respect to the position vector $\boldsymbol{x}$ is defined as

$$
\frac{\partial \delta \boldsymbol{\xi}}{\partial \boldsymbol{x}}:=\delta \boldsymbol{\xi}_{, x} \otimes \boldsymbol{e}_{x}^{I}+\delta \boldsymbol{\xi}_{, y} \otimes \boldsymbol{e}_{y}^{I}+\delta \boldsymbol{\xi}_{, z} \otimes \boldsymbol{e}_{z}^{I} .
$$

The partial derivatives $\delta \boldsymbol{\xi}_{, i}$ of the virtual displacement field with respect to the coordinates $i=x, y, z$ can therefore be extracted by contracting the last tensor slot of the partial derivative with the basis vectors $\boldsymbol{e}_{i}^{I}$ :

$$
\delta \boldsymbol{\xi}_{, x}=\frac{\partial \delta \boldsymbol{\xi}}{\partial \boldsymbol{x}} \cdot \boldsymbol{e}_{x}^{I}, \quad \delta \boldsymbol{\xi}_{, y}=\frac{\partial \delta \boldsymbol{\xi}}{\partial \boldsymbol{x}} \cdot \boldsymbol{e}_{y}^{I}, \quad \delta \boldsymbol{\xi}_{, z}=\frac{\partial \delta \boldsymbol{\xi}}{\partial \boldsymbol{x}} \cdot \boldsymbol{e}_{z}^{I},
$$

where the dot denotes the contraction. For a second-order tensor $A \in \mathbb{E}^{3} \otimes \mathbb{E}^{3}$ and vectors $\boldsymbol{u}, \boldsymbol{v} \in \mathbb{E}^{3}$, the relation $(\boldsymbol{A} \cdot \boldsymbol{v}) \cdot \boldsymbol{u}=\boldsymbol{u} \cdot(\boldsymbol{A} \cdot \boldsymbol{v})=\boldsymbol{A}:(\boldsymbol{u} \otimes \boldsymbol{v})$ holds. Using this relation together with (26), the terms involving the stress vectors $\boldsymbol{t}_{i}$ in (24) are reformulated as

$$
\begin{aligned}
\delta \boldsymbol{\xi}_{, x} \cdot \boldsymbol{t}_{x}+\delta \boldsymbol{\xi}_{, y} \cdot \boldsymbol{t}_{y}+\delta \boldsymbol{\xi}_{, z} \cdot \boldsymbol{t}_{z} & \\
& =\left(\frac{\partial \delta \boldsymbol{\xi}}{\partial \boldsymbol{x}} \cdot \boldsymbol{e}_{x}^{I}\right) \cdot \boldsymbol{t}_{x}+\left(\frac{\partial \delta \boldsymbol{\xi}}{\partial \boldsymbol{x}} \cdot \boldsymbol{e}_{y}^{I}\right) \cdot \boldsymbol{t}_{y}+\left(\frac{\partial \delta \boldsymbol{\xi}}{\partial \boldsymbol{x}} \cdot \boldsymbol{e}_{z}^{I}\right) \cdot \boldsymbol{t}_{z}=\frac{\partial \delta \boldsymbol{\xi}}{\partial \boldsymbol{x}}: \boldsymbol{\sigma},
\end{aligned}
$$

where the stress tensor $\sigma$ emerges as being the second-order Euclidean tensor field

$$
\boldsymbol{\sigma}(\boldsymbol{x}):=\boldsymbol{t}_{x}(\boldsymbol{x}) \otimes \boldsymbol{e}_{x}^{I}+\boldsymbol{t}_{y}(\boldsymbol{x}) \otimes \boldsymbol{e}_{y}^{I}+\boldsymbol{t}_{z}(\boldsymbol{x}) \otimes \boldsymbol{e}_{z}^{I} .
$$

The stress tensor field (28) as the tensor field over the deformed configuration $\Omega$ is called Cauchy stress. Using (27), the virtual work of the stress (24) can now be written in the form

$$
\delta W^{\mathrm{s}}=\int_{\Omega}\left(\delta \boldsymbol{\xi} \cdot \boldsymbol{c}-\frac{\partial \delta \boldsymbol{\xi}}{\partial \boldsymbol{x}}: \boldsymbol{\sigma}\right) \mathrm{d} v .
$$

Comparing the virtual work (29) with the possible force representations (4), we immediately recognize that the stress contributes with volume measures of order zero, $\mathrm{d} \boldsymbol{F}^{0}=\boldsymbol{c} \mathrm{d} v$, and order one, $\mathrm{d} \boldsymbol{F}^{1}=-\sigma \mathrm{d} v$. Even though the derivation of (29) makes use of cartesian coordinates for the parametrization of the domain $\Omega$, the virtual work of the stress (29) includes only Euclidean vector and tensor 
fields together with tensor operations. Consequently, (29) is independent of any choice of coordinates and can therefore be claimed to be coordinate-free. Note that definitions (25) and (28) rely on cartesian coordinates. For curvilinear coordinates, these definitions have to be revisited and require the concepts of coand contravariant basis vectors [Başar and Weichert 2000; Green and Zerna 1954; Ogden 1997], which goes beyond the scope of this paper. However, and herein lies the strength of a variational formulation, since the virtual work is invariant with respect to the parametrization of the appearing integral, (24) can also be applied to curvilinear coordinates. Without introducing partial derivatives or divergence operators for curvilinear coordinates, we will demonstrate in the Appendix how to derive the local equilibrium equations in cylindrical coordinates, by starting from (24) instead of (29). For spaces more general than $\mathbb{E}^{3}$, as for example manifolds, the partial derivative in (29) is not defined anymore and has to be exchanged with a covariant derivative, as discussed in [Eugster 2015a; 2015b; Segev 1986].

\section{Symmetry of the stress tensor}

In this section, we prove the symmetry of the stress tensor. Our approach basically follows the strategy proposed by Germain $[1972 ; 1973 b]$ and can be regarded as a detailed version of it. According to Definition 3, stresses contribute to the internal forces of the body. If now the stresses are the only internal force contributions, they also form the totality of internal forces and as such they have to satisfy the variational law of interaction (Axiom 2) in their entirety. The following proof is nothing else but the evaluation of the variational law of interaction for the stresses. Note that the proof will be carried out by exclusively using the variational law of interaction, meaning that external forces are nowhere needed in the argumentation up to the point at which the existence of the stress tensor is addressed. We start with the variational law of interaction (6), by which the virtual work $\delta W^{\mathrm{s}}$ of the stress has to satisfy

$$
\delta W^{\mathrm{s}}=0 \quad \text { for all } \delta \boldsymbol{\xi}_{K, \text { rig }}(\boldsymbol{x})= \begin{cases}\delta \boldsymbol{\xi}_{\mathrm{rig}}(\boldsymbol{x}) & \text { for } \boldsymbol{x} \in K \\ 0 & \text { for } \boldsymbol{x} \notin K\end{cases}
$$

for any open set $K \subseteq \Omega$ with rigid virtual displacements $\delta \xi_{\text {rig }}$ as defined in (3). Equation (30) implies additional conditions on the force quantities $\boldsymbol{c}$ and $\boldsymbol{t}_{i}$ in (24), which are elaborated in the following and which will lead to the symmetry conditions in question. Combining (30) and (24) yields

$$
0=\int_{K}\left(\delta \boldsymbol{\xi}_{\mathrm{rig}} \cdot \boldsymbol{c}-\delta \boldsymbol{\xi}_{\mathrm{rig}, x} \cdot \boldsymbol{t}_{x}-\delta \boldsymbol{\xi}_{\mathrm{rig}, y} \cdot \boldsymbol{t}_{y}-\delta \boldsymbol{\xi}_{\mathrm{rig}, z} \cdot \boldsymbol{t}_{z}\right) \mathrm{d} v \quad \text { for all } K \text { and all } \delta \boldsymbol{\xi}_{\mathrm{rig}}
$$


The rigid virtual displacement fields $\delta \boldsymbol{\xi}_{\text {rig }}$ are taken from (3), and their partial derivatives $\delta \xi_{\text {rig, }, i}$ for $i=x, y, z$ are accordingly calculated as

$$
\delta \boldsymbol{\xi}_{\text {rig }}(\boldsymbol{x})=\delta \boldsymbol{r}_{O}+\delta \boldsymbol{\phi} \times \boldsymbol{x} \quad \text { and } \quad \delta \boldsymbol{\xi}_{\mathrm{rig}, i}(\boldsymbol{x})=\delta \boldsymbol{\phi} \times \boldsymbol{x}_{, i} .
$$

Inserting (32) into (31), one obtains the variational condition

$$
\begin{array}{r}
0=\int_{K}\left(\left(\delta \boldsymbol{r}_{O}+\delta \boldsymbol{\phi} \times \boldsymbol{x}\right) \cdot \boldsymbol{c}-\left(\delta \boldsymbol{\phi} \times \boldsymbol{x}_{, x}\right) \cdot \boldsymbol{t}_{x}-\left(\delta \boldsymbol{\phi} \times \boldsymbol{x}_{, y}\right) \cdot \boldsymbol{t}_{y}-\left(\delta \boldsymbol{\phi} \times \boldsymbol{x}_{, z}\right) \cdot \boldsymbol{t}_{z}\right) \mathrm{d} v \\
\text { for all } K, \text { all } \delta \boldsymbol{r}_{O}, \text { and all } \delta \boldsymbol{\phi} .
\end{array}
$$

We use the relation $(\delta \phi \times z) \cdot \boldsymbol{f}=\delta \boldsymbol{\phi} \cdot(\boldsymbol{z} \times \boldsymbol{f})$ to move the quantities $\delta \boldsymbol{r}_{O}$ and $\delta \boldsymbol{\phi}$ in front of the integral and obtain

$0=\delta \boldsymbol{r}_{O} \cdot \int_{K} \boldsymbol{c} \mathrm{d} v+\delta \boldsymbol{\phi} \cdot \int_{K}\left(\boldsymbol{x} \times \boldsymbol{c}-\boldsymbol{x}_{, x} \times \boldsymbol{t}_{x}-\boldsymbol{x}_{, y} \times \boldsymbol{t}_{y}-\boldsymbol{x}_{, z} \times \boldsymbol{t}_{z}\right) \mathrm{d} v$

$$
\text { for all } K \text {, all } \delta \boldsymbol{r}_{O} \text {, and all } \delta \boldsymbol{\phi} \text {. }
$$

The variational law of interaction for the stresses has now been brought into a form which allows one to evaluate the variations $\delta \boldsymbol{r}_{O}$ and $\delta \boldsymbol{\phi}$, and to carry out a subsequent localization step based on the fundamental lemma of calculus of variations.

We start to carry out the variation for all $\delta \boldsymbol{r}_{O}$, while making the specific choice $\delta \boldsymbol{\phi} \equiv 0$. Consequently, (34) reduces to

$$
0=\delta \boldsymbol{r}_{O} \cdot \int_{K} \boldsymbol{c} \mathrm{d} v \quad \text { for all } K \text { and all } \delta \boldsymbol{r}_{O} .
$$

Since (35) has to hold for all $\delta \boldsymbol{r}_{O}$, we obtain

$$
0=\int_{K} c \mathrm{~d} v \text { for all } K
$$

If now $\boldsymbol{c}$ is continuous on a neighborhood $K$ of $\boldsymbol{x}$, then (36) can be localized, which yields

$$
\boldsymbol{c}(\boldsymbol{x})=0 .
$$

The question whether $\boldsymbol{c}$ is continuous at $\boldsymbol{x}$ can not be answered here, i.e., by the variational law of interaction alone. The continuity of $\boldsymbol{c}$ strongly depends on the external forces that are applied on the body, as well as on the constitutive laws that still have to be specified for both the internal and external forces. In other words, the continuity of $\boldsymbol{c}$ involves the principle of virtual work (Axiom 1) because of the external forces, together with the choice of the constitutive laws, and can be checked only after the entire continuum problem has been solved.

In the case that $\boldsymbol{c}$ is continuous at $\boldsymbol{x}$, we have $\boldsymbol{c}=0$ by (37); hence, $\boldsymbol{c}_{x}+\boldsymbol{c}_{y}+\boldsymbol{c}_{z}=0$ by (23). The latter means that the three volume forces $\boldsymbol{c}_{i}$ cancel out each other on the considered volume element and can therefore be disregarded in the ansatz (13). As a consequence, the ansatz (13) simplifies to $\Delta \boldsymbol{G}_{x}\left(x_{i}\right)=\Delta \boldsymbol{F}_{x}\left(x_{i}\right)$ and 
$\Delta \boldsymbol{H}_{x}\left(x_{i}+\Delta x\right)=-\Delta \boldsymbol{F}_{x}\left(x_{i}\right)$, and in the same fashion for the directions $y$ and $z$, with which we have proven that action equals reaction applies for the stress vectors $\boldsymbol{t}_{i}$.

For the upcoming evaluation of (34) with respect to the variations $\delta \boldsymbol{\phi}$, we assume continuity of $\boldsymbol{c}$, that is, $\boldsymbol{c}=0$. As a consequence, the variational problem (34) reduces to

$$
0=\delta \phi \cdot \int_{K}\left(-\boldsymbol{x}_{, x} \times \boldsymbol{t}_{x}-\boldsymbol{x}_{, y} \times \boldsymbol{t}_{y}-\boldsymbol{x}_{, z} \times \boldsymbol{t}_{z}\right) \mathrm{d} v \quad \text { for all } K \text { and all } \delta \phi .
$$

Since (38) has to hold for all $\delta \boldsymbol{\phi}$, we obtain

$$
0=\int_{K}\left(-\boldsymbol{x}_{, x} \times \boldsymbol{t}_{x}-\boldsymbol{x}_{, y} \times \boldsymbol{t}_{y}-\boldsymbol{x}_{, z} \times \boldsymbol{t}_{z}\right) \mathrm{d} v \text { for all } K .
$$

By using the same arguments for the localizing as above, we obtain the symmetry conditions for the stress vectors, which are

$$
\boldsymbol{x}_{, x} \times \boldsymbol{t}_{x}(\boldsymbol{x})+\boldsymbol{x}_{, y} \times \boldsymbol{t}_{y}(\boldsymbol{x})+\boldsymbol{x}_{, z} \times \boldsymbol{t}_{z}(\boldsymbol{x})=0 .
$$

They again hold only for stress vector fields $\boldsymbol{t}_{i}$ which are continuous at $\boldsymbol{x}$. This continuity can again only be checked after the evaluation of the principle of virtual work together with the constitutive laws. A discontinuity appears for instance when an external surface force is applied on a surface inside the body. The same holds true for line forces and point forces. In these cases, continuity can be assumed at least piecewise. Another example showing that the limits of $\boldsymbol{C}_{i}$ and $\boldsymbol{T}_{i}$ in (16) do not even have to exist, at least not uniquely, are the force interactions within a rigid body.

The symmetry of the stress tensor (28) is proven in the same manner as we have shown the symmetry condition for the stress vectors, with the only difference that we start now with the virtual work contribution in the form (29), instead of (24), with vanishing volume force $\boldsymbol{c}=0$. In (29) and (30), the partial derivative of the rigid virtual displacement field with respect to the position vector $\boldsymbol{x}$ is needed, which is by (3) the variation $\delta \boldsymbol{\phi} \times=\delta \tilde{\boldsymbol{\phi}}=\tilde{\boldsymbol{\phi}}_{, \varepsilon}\left(\varepsilon_{0}\right)$ of the skew-symmetric linear map $\tilde{\boldsymbol{\phi}}(\varepsilon)$ as introduced in the text below of (3). Similar to (38), the variational law of interaction implies the condition

$$
0=-\int_{K} \frac{\partial \delta \boldsymbol{\xi}_{\mathrm{rig}}}{\partial \boldsymbol{x}}: \sigma \mathrm{d} v=-\int_{K} \delta \tilde{\boldsymbol{\phi}}: \boldsymbol{\sigma} \mathrm{d} v \quad \text { for all } K \text { and all } \delta \boldsymbol{\phi} .
$$

For a continuous stress tensor field $\sigma,(41)$ can be localized to provide the variational condition

$$
\delta \tilde{\boldsymbol{\phi}}: \sigma=0 \text { for all } \delta \boldsymbol{\phi} .
$$

Now, for $\boldsymbol{S}, \boldsymbol{W} \in \mathbb{E}^{3} \otimes \mathbb{E}^{3}$ with $\boldsymbol{S}$ being symmetric and $\boldsymbol{W}$ being skew-symmetric, it can be shown ${ }^{14}$ that $\boldsymbol{S}: \boldsymbol{W}=0$. Since $\delta \tilde{\boldsymbol{\phi}}$ is skew-symmetric, the only nontrivial

${ }^{14} \boldsymbol{S}: \boldsymbol{W}=\operatorname{tr}\left(\boldsymbol{S}^{\mathrm{T}} \cdot \boldsymbol{W}\right)=\operatorname{tr}\left(\boldsymbol{W} \cdot \boldsymbol{S}^{\mathrm{T}}\right)=\boldsymbol{W}^{\mathrm{T}}: \boldsymbol{S}^{\mathrm{T}}=\boldsymbol{S}^{\mathrm{T}}: \boldsymbol{W}^{\mathrm{T}}=-\boldsymbol{S}: \boldsymbol{W}$ implies $\boldsymbol{S}: \boldsymbol{W}=0$. 
solution for $\sigma$ to guarantee (42) is to be symmetric, which proves the symmetry of the stress tensor

$$
\sigma=\sigma^{\mathrm{T}}
$$

Depending on the specific application, one might choose between the representations (24) and (29) of the virtual work of stress. The same applies for the symmetry conditions, which may be stated either in terms of the stress vectors (40) or in terms of the stress tensor (43). Which one is preferred is often a matter of taste.

For generalized continua as for instance micromorphic continua of degree one [Germain 1973b], the stress tensor obtained from Definition 3 corresponds to the socalled intrinsic stress, which is always symmetric. By the introduction of additional degrees of freedom, as used to describe some microstructure, the nonsymmetric microstress tensor appears as a coupling stress between the micro and the macro levels. ${ }^{15}$ Since the sum of these two stress tensors appears in relation with the surface forces, this sum is commonly called the Cauchy stress tensor, which is in general a nonsymmetric second-order tensor.

\section{Transformation properties of stress}

As mentioned in Section 3, the invariance of the virtual work provides the required transformation properties of the appearing force quantities, when different integral parametrizations or coordinate representations are used. Applying this invariance to the virtual work of the stress (24) or (29), various stress representations can be derived. Without the notion of real work or power, we are able to obtain the work conjugate stress representations [Başar and Weichert 2000; Macvean 1968] from the virtual work alone. Since there is a wealth of possible stress and coordinate representations, we show here just a selection of three important transformation properties. The first is a reparametrization of the integral in (24) with curvilinear coordinates. The second is a reparametrization of the integral in (29) with respect to a new set of position vectors addressing a reference configuration. The last transformation property is obtained for the coordinate representation of (29) when using cartesian coordinates only. All transformation properties are derived for continuous fields, i.e., for $c=0$, with the virtual work contribution of the stress

$$
\delta W^{\mathrm{s}}=-\int_{\Omega}\left(\delta \boldsymbol{\xi}_{, x} \cdot \boldsymbol{t}_{x}+\delta \boldsymbol{\xi}_{, y} \cdot \boldsymbol{t}_{y}+\delta \boldsymbol{\xi}_{, z} \cdot \boldsymbol{t}_{z}\right) \mathrm{d} v=-\int_{\Omega} \frac{\partial \delta \boldsymbol{\xi}}{\partial \boldsymbol{x}}: \boldsymbol{\sigma} \mathrm{d} v,
$$

satisfying the symmetry conditions (40) and (43).

The first integral in (44) is formulated in the deformed configuration and parametrized by cartesian coordinates ${ }_{I} \boldsymbol{x}=(x, y, z)^{\mathrm{T}}$. For a set of curvilinear coordinates

${ }^{15}$ Note that since in this context the micro level is also described by a continuous field, it has to be understood in a way different from the micro level as used in this paper. 
$(p, q, r)^{\mathrm{T}}$ as, e.g., cylindrical coordinates, there exists at least a local invertible map $\varphi$ relating the cartesian with the curvilinear coordinates by

$$
{ }_{I} \boldsymbol{x}=\boldsymbol{\varphi}(p, q, r)=\left(\begin{array}{c}
x(p, q, r) \\
y(p, q, r) \\
z(p, q, r)
\end{array}\right) \quad \text { and } \quad\left(\begin{array}{c}
p \\
q \\
r
\end{array}\right)=\boldsymbol{\varphi}^{-1}\left({ }_{I} \boldsymbol{x}\right)=\left(\begin{array}{c}
p\left({ }_{I} \boldsymbol{x}\right) \\
q\left({ }_{I} \boldsymbol{x}\right) \\
r\left({ }_{I} \boldsymbol{x}\right)
\end{array}\right) .
$$

Note that curvilinear coordinates cannot be related to a position vector $\boldsymbol{x}$ in the sense of (1), where each of the coordinates in ${ }_{I} x$ corresponds to one of the components of the position vector $\boldsymbol{x}$. The volume element in (44) can be expressed in new coordinates as

$\mathrm{d} v=\mathrm{d} x \mathrm{~d} y \mathrm{~d} z=J \mathrm{~d} p \mathrm{~d} q \mathrm{~d} r=J \mathrm{~d} \hat{v}$

$$
\text { with } J=\operatorname{det}(D \varphi)(p, q, r) \text { and } \mathrm{d} \hat{v}=\mathrm{d} p \mathrm{~d} q \mathrm{~d} r,
$$

where $D \varphi$ denotes the Jacobian matrix containing the partial derivatives of $\varphi$. To still integrate over the same set in the Euclidean space, the domain of integration transforms to $\widehat{\Omega}=\varphi^{-1}(\Omega)$. Furthermore, we introduce the virtual displacement field in curvilinear coordinates $\delta \hat{\boldsymbol{\xi}}$, such that the identity

$$
\delta \boldsymbol{\xi}\left({ }_{I} \boldsymbol{x}\right)=\delta \hat{\boldsymbol{\xi}}\left(\boldsymbol{\varphi}^{-1}\left({ }_{I} \boldsymbol{x}\right)\right)=\delta \hat{\boldsymbol{\xi}}\left(p\left({ }_{I} \boldsymbol{x}\right), q\left({ }_{I} \boldsymbol{x}\right), r\left({ }_{I} \boldsymbol{x}\right)\right)
$$

holds. Due to (45) and (47) together with the chain rule, we can reformulate the virtual work density in (44) now as

$$
\begin{aligned}
& \delta \boldsymbol{\xi}_{, x} \cdot \boldsymbol{t}_{x}+ \delta \boldsymbol{\xi}_{, y} \cdot \boldsymbol{t}_{y}+\delta \boldsymbol{\xi}_{, z} \cdot \boldsymbol{t}_{z} \\
&=\left(\delta \hat{\boldsymbol{\xi}}_{, p} \frac{\partial p}{\partial x}+\delta \hat{\boldsymbol{\xi}}_{, q} \frac{\partial q}{\partial x}+\delta \hat{\boldsymbol{\xi}}_{, r} \frac{\partial r}{\partial x}\right) \cdot \boldsymbol{t}_{x}+\left(\delta \hat{\boldsymbol{\xi}}_{, p} \frac{\partial p}{\partial y}+\delta \hat{\boldsymbol{\xi}}_{, q} \frac{\partial q}{\partial y}+\delta \hat{\boldsymbol{\xi}}_{, r} \frac{\partial r}{\partial y}\right) \cdot \boldsymbol{t}_{y}+\left(\delta \hat{\boldsymbol{\xi}}_{, p} \frac{\partial p}{\partial z}+\delta \hat{\boldsymbol{\xi}}_{, q} \frac{\partial q}{\partial z}+\delta \hat{\boldsymbol{\xi}}_{, r} \frac{\partial r}{\partial z}\right) \cdot \boldsymbol{t}_{z} \\
&=\delta \hat{\boldsymbol{\xi}}_{, p} \cdot\left(\frac{\partial p}{\partial x} \boldsymbol{t}_{x}+\frac{\partial p}{\partial y} \boldsymbol{t}_{y}+\frac{\partial p}{\partial z} \boldsymbol{t}_{z}\right)+\delta \hat{\boldsymbol{\xi}}_{, q} \cdot\left(\frac{\partial q}{\partial x} \boldsymbol{t}_{x}+\frac{\partial q}{\partial y} \boldsymbol{t}_{y}+\frac{\partial q}{\partial z} \boldsymbol{t}_{z}\right) \\
&+\delta \hat{\boldsymbol{\xi}}, r_{,} \cdot\left(\frac{\partial r}{\partial x} \boldsymbol{t}_{x}+\frac{\partial r}{\partial y} \boldsymbol{t}_{y}+\frac{\partial r}{\partial z} \boldsymbol{t}_{z}\right) \\
&=\delta \hat{\boldsymbol{\xi}}_{, p} \cdot \hat{\boldsymbol{t}}_{p}+\delta \hat{\boldsymbol{\xi}}_{, q} \cdot \hat{\boldsymbol{t}}_{q}+\delta \hat{\boldsymbol{\xi}}, r_{,} \cdot \hat{\boldsymbol{t}}_{r},
\end{aligned}
$$

where we have introduced the new stress vectors

$$
\begin{aligned}
& \hat{\boldsymbol{t}}_{p}=\frac{\partial p}{\partial x} \boldsymbol{t}_{x}+\frac{\partial p}{\partial y} \boldsymbol{t}_{y}+\frac{\partial p}{\partial z} \boldsymbol{t}_{z}, \\
& \hat{\boldsymbol{t}}_{q}=\frac{\partial q}{\partial x} \boldsymbol{t}_{x}+\frac{\partial q}{\partial y} \boldsymbol{t}_{y}+\frac{\partial q}{\partial z} \boldsymbol{t}_{z}, \\
& \hat{\boldsymbol{t}}_{r}=\frac{\partial r}{\partial x} \boldsymbol{t}_{x}+\frac{\partial r}{\partial y} \boldsymbol{t}_{y}+\frac{\partial r}{\partial z} \boldsymbol{t}_{z} .
\end{aligned}
$$


Using (48) together with (46) in (44), we observe the virtual work of the stress to be of the same form in cartesian and curvilinear coordinates:

$$
\begin{aligned}
\delta W^{\mathrm{s}}=-\int_{\Omega}\left(\delta \boldsymbol{\xi}_{, x} \cdot \boldsymbol{t}_{x}+\delta \boldsymbol{\xi}_{, y} \cdot \boldsymbol{t}_{y}\right. & \left.+\delta \boldsymbol{\xi}_{, z} \cdot \boldsymbol{t}_{z}\right) \mathrm{d} v \\
& =-\int_{\widehat{\Omega}}\left(\delta \hat{\boldsymbol{\xi}}_{, p} \cdot \hat{\boldsymbol{t}}_{p}+\delta \hat{\boldsymbol{\xi}}_{, q} \cdot \hat{\boldsymbol{t}}_{q}+\delta \hat{\boldsymbol{\xi}}_{, r} \cdot \hat{\boldsymbol{t}}_{r}\right) J \mathrm{~d} \hat{v}
\end{aligned}
$$

Note that the determinant of the Jacobian is $J=1$ for cartesian coordinates. Alternatively, we can consider $J \mathrm{~d} \hat{v}$ to be the Riemannian volume element, where $J$ corresponds to the square root of the first fundamental form of the mapping $\varphi$ [Kühnel 2013; Lee 2013]. Moreover, as a direct consequence of (50), it follows that the symmetry condition (40) applies in the same form when $(x, y, z)$ are curvilinear coordinates.

The integral in the rightmost expression of (44) is parametrized by the position vector $\boldsymbol{x}$ of the deformed configuration. Consequently, the domain of integration $\Omega$ changes for different configurations. It is often convenient to introduce a special configuration $\bar{\Omega}_{0}$, called the reference configuration, in which certain information, as for example the stress state and the dimensions of the body, are available. The position vectors $\boldsymbol{X}$ of the material points in the reference configuration are in a bijective relation with the position vectors $x$ in the deformed configuration; i.e., $\varphi: \bar{\Omega}_{0} \rightarrow \bar{\Omega}$ with $X \mapsto x=\varphi(X)$. Note, in contrast to (45), that the function $\varphi$ is here a mapping between subsets of $\mathbb{E}^{3}$ but not of $\mathbb{R}^{3}$. Using the mapping $\varphi$, we can express the integral in (44) with respect to the reference configuration $\bar{\Omega}_{0}=\varphi^{-1}(\bar{\Omega})$. This yields

$$
\mathrm{d} v=J \mathrm{~d} V \quad \text { with } J=\operatorname{det} \boldsymbol{F},
$$

where $\boldsymbol{F}=\partial \boldsymbol{\varphi} / \partial \boldsymbol{X}$ is the deformation gradient. The virtual displacement field over the reference configuration $\delta \boldsymbol{\Xi}: \bar{\Omega}_{0} \rightarrow \mathbb{E}^{3}$ is under the mapping $\varphi$ obtained as

$$
\delta \boldsymbol{\xi}(\boldsymbol{x})=\delta \boldsymbol{\Xi}\left(\boldsymbol{\varphi}^{-1}(\boldsymbol{x})\right) .
$$

Using (51) and (52) together with the chain rule, the last expression in (44) can be reformulated as

$$
\begin{aligned}
\delta W^{\mathrm{s}}=-\int_{\Omega} \frac{\partial \delta \boldsymbol{\xi}}{\partial \boldsymbol{x}}: \boldsymbol{\sigma} \mathrm{d} v=-\int_{\Omega_{0}}\left(\frac{\partial \delta \boldsymbol{\Xi}}{\partial \boldsymbol{X}} \cdot \frac{\partial \boldsymbol{\varphi}^{-1}}{\partial \boldsymbol{x}}\right): \boldsymbol{\sigma} J \mathrm{~d} V \\
=-\int_{\Omega_{0}}\left(\frac{\partial \delta \boldsymbol{\Xi}}{\partial \boldsymbol{X}} \cdot \boldsymbol{F}^{-1}\right): \boldsymbol{\sigma} J \mathrm{~d} V,
\end{aligned}
$$

where $\boldsymbol{F}^{-1}=\partial \boldsymbol{\varphi}^{-1} / \partial \boldsymbol{x}$ denotes the inverse of the deformation gradient. With the relation $(\boldsymbol{A} \cdot \boldsymbol{B}): \boldsymbol{C}=\boldsymbol{A}:\left(\boldsymbol{C} \cdot \boldsymbol{B}^{\mathrm{T}}\right)$, we rewrite (53) as

$$
\delta W^{\mathrm{s}}=-\int_{\Omega_{0}} \frac{\partial \delta \boldsymbol{\Xi}}{\partial \boldsymbol{X}}:\left(J \boldsymbol{\sigma} \cdot \boldsymbol{F}^{-\mathrm{T}}\right) \mathrm{d} V=-\int_{\Omega_{0}} \frac{\partial \delta \boldsymbol{\Xi}}{\partial \boldsymbol{X}}: \boldsymbol{P} \mathrm{d} V,
$$


in which the new stress representation, the first Piola-Kirchhoff stress,

$$
\boldsymbol{P}:=J \boldsymbol{\sigma} \cdot \boldsymbol{F}^{-\mathrm{T}}
$$

has been identified. Further manipulation of (53) leads to other stress representations [Bonet and Wood 1997]. The transformation of the virtual work expression guarantees the kinematical and the force quantities to always be work conjugate.

The last important transformation property is obtained when the stress vectors and the virtual displacement field are represented with respect to orthonormal frames. In addition to the $I$-system, we introduce the $B$-system $\left(\boldsymbol{e}_{x}^{B}, \boldsymbol{e}_{y}^{B}, \boldsymbol{e}_{z}^{B}\right)$ which is rotated with respect to the $I$-system. The position vector $\boldsymbol{x}$ represented in the $B$-system is given by the tuple ${ }_{B} \boldsymbol{x}=(\hat{x}, \hat{y}, \hat{z})^{\mathrm{T}}$. Since the two coordinate systems are rotated against each other, the coordinate representations of vectors $\boldsymbol{u} \in \mathbb{E}^{3}$ are related by

$$
{ }_{I} \boldsymbol{u}=\boldsymbol{A}_{I B B} \boldsymbol{u},
$$

with the transformation matrix $\boldsymbol{A}_{I B}$ being orthogonal, i.e., $\boldsymbol{A}_{I B}^{\mathrm{T}} \boldsymbol{A}_{I B}=\boldsymbol{A}_{I B} \boldsymbol{A}_{I B}^{\mathrm{T}}=\boldsymbol{I}$. The inverse of the transformation matrix $\boldsymbol{A}_{I B}$ is denoted as $\boldsymbol{A}_{B I}=\boldsymbol{A}_{I B}^{-1}=\boldsymbol{A}_{I B}^{\mathrm{T}}$. Furthermore, the coordinate representation of the virtual displacement field and its partial derivative in the $I$-system are

$$
\begin{aligned}
&{ }_{I} \delta \boldsymbol{\xi}\left({ }_{I} \boldsymbol{x}\right)=\left(\begin{array}{l}
\delta \xi_{x}^{I}\left({ }_{I} \boldsymbol{x}\right) \\
\delta \xi_{y}^{I}\left({ }_{I} \boldsymbol{x}\right) \\
\delta \xi_{z}^{I}\left({ }_{I} \boldsymbol{x}\right)
\end{array}\right), \\
& \frac{\partial_{I} \delta \boldsymbol{\xi}}{\partial_{I} \boldsymbol{x}} \equiv\left(\begin{array}{llll}
{ }_{I} \delta \boldsymbol{\xi}_{, x} & { }_{I} \delta \boldsymbol{\xi}_{, y} & { }_{I} \delta \boldsymbol{\xi}_{, z}
\end{array}\right)=\left(\begin{array}{lll}
\delta \xi_{x, x}^{I} & \delta \xi_{x, y}^{I} & \delta \xi_{x, z}^{I} \\
\delta \xi_{y, x}^{I} & \delta \xi_{y, y}^{I} & \delta \xi_{y, z}^{I} \\
\delta \xi_{z, x}^{I} & \delta \xi_{z, y}^{I} & \delta \xi_{z, z}^{I}
\end{array}\right) .
\end{aligned}
$$

By exchanging $(x, y, z)$ with $(\hat{x}, \hat{y}, \hat{z})$ and $I$ with $B$ in (57), we obtain the coordinate representation in the $B$-system. The same holds for the stress tensor $\sigma$, whose coordinate representation in the $I$-system is, due to (28), the matrix

$$
{ }_{I} \boldsymbol{\sigma}:=\left(\begin{array}{lll}
{ }_{I} \boldsymbol{t}_{x} & { }_{I} \boldsymbol{t}_{y}{ }_{I} \boldsymbol{t}_{z}
\end{array}\right)=\left(\begin{array}{ccc}
\sigma_{x x}^{I} & \tau_{x y}^{I} & \tau_{x z}^{I} \\
\tau_{y x}^{I} & \sigma_{y y}^{I} & \tau_{y z}^{I} \\
\tau_{z x}^{I} & \tau_{z y}^{I} & \sigma_{z z}^{I}
\end{array}\right) .
$$

The coordinate representation of the rightmost term in (44) is further transformed by using (56) and the chain rule to give

$$
\begin{array}{r}
\delta W^{\mathrm{s}=}=-\int_{\Omega} \frac{\partial_{I} \delta \xi}{\partial_{I} \boldsymbol{x}}:{ }_{I} \boldsymbol{\sigma} \mathrm{d} v=-\int_{\widehat{\Omega}}\left(\frac{\partial\left(\boldsymbol{A}_{I B B} \delta \boldsymbol{\xi}\right)}{\partial_{B} \boldsymbol{x}} \frac{\partial_{B} \boldsymbol{x}}{\partial_{I} \boldsymbol{x}}\right):{ }_{I} \boldsymbol{\sigma} \mathrm{d} \hat{v} \\
=-\int_{\widehat{\Omega}}\left(\boldsymbol{A}_{I B} \frac{\partial_{B} \delta \boldsymbol{\xi}}{\partial_{B} \boldsymbol{x}} \boldsymbol{A}_{I B}^{\mathrm{T}}\right):{ }_{I} \boldsymbol{\sigma} \mathrm{d} \hat{v}
\end{array}
$$


where $\widehat{\Omega}$ denotes the open set of the rotated domain with the volume element $\mathrm{d} \hat{v}=$ $\mathrm{d} \hat{x} \mathrm{~d} \hat{y} \mathrm{~d} \hat{z}$. Using the symmetry of the double contraction and twice the relation $(\boldsymbol{A} \boldsymbol{B}): \boldsymbol{C}=\boldsymbol{A}:\left(\boldsymbol{C} \boldsymbol{B}^{\mathrm{T}}\right)$, which holds also for matrices, (59) becomes

$$
\begin{aligned}
\delta W^{\mathrm{S}}=-\int_{\widehat{\Omega}} \frac{\partial_{B} \delta \xi}{\partial_{B} \boldsymbol{x}}:\left(\boldsymbol{A}_{I B}^{\mathrm{T}} \boldsymbol{\sigma} \boldsymbol{\sigma} \boldsymbol{A}_{I B}\right) \mathrm{d} \hat{v}=-\int_{\widehat{\Omega}} \frac{\partial_{B} \delta \xi}{\partial_{B} \boldsymbol{x}} & :\left(\boldsymbol{A}_{B I I} \boldsymbol{\sigma} \boldsymbol{A}_{B I}^{\mathrm{T}}\right) \mathrm{d} \hat{v} \\
& =-\int_{\widehat{\Omega}} \frac{\partial_{B} \delta \xi}{\partial_{B} \boldsymbol{x}}:{ }_{B} \boldsymbol{\sigma} \mathrm{d} \hat{v},
\end{aligned}
$$

with which the transformation rule of the coordinate representation of the stress tensor is identified to be

$$
{ }_{B} \sigma=A_{B I I} \sigma A_{B I}^{\mathrm{T}}
$$

The transformation rule (61) is of vital importance in the derivation of Mohr's circle, which is a graphical method to find the principal axis of the stress tensor $\sigma$.

\section{Boundary value problem}

Starting from the principle of virtual work (Axiom 1), we derive in this section the partial differential equations together with their boundary conditions, which describe a deformable body under the force interactions assumed as follows. As internal forces of the deformed configuration $\bar{\Omega}$, we allow solely stresses $\sigma$ that are short-range forces according to Definition 3. Any other kinds of internal forces, as for example force interactions of nonneighboring volume elements by long-range forces, are excluded. For the external forces, we assume that they either contribute as volume forces $f_{v} \mathrm{~d} v$ in the interior $\Omega$ of $\bar{\Omega}$, or as surface forces $f_{a} \mathrm{~d} a$ on the boundary $\partial \Omega$ of $\bar{\Omega}$. The following derivation corresponds de facto with the reverse direction of the approach presented by [Clebsch 1862; Lamé 1852].

To derive the boundary value problem, the virtual work of internal forces has to be integrated by parts, for which we use the following integral theorem of $\mathbb{R}^{3}$. Let $\Omega \subset \mathbb{R}^{3}$ be an open subset of $\mathbb{R}^{3}, \bar{\Omega}$ be the closure of $\Omega$, and $\partial \Omega=\bar{\Omega} \backslash \Omega$ be the boundary of $\Omega$. For a function $f(x, y, z)$ on $\Omega$, integration holds in the form

$$
\left.\int_{\Omega} \frac{\partial f}{\partial x} \mathrm{~d} v=\int_{\bar{\Omega}} \frac{\partial f}{\partial x} \mathrm{~d} v=\int_{\partial \Omega} f n^{x} \mathrm{~d} a \quad \text { (same for } y \text { and } z\right),
$$

where ${ }_{I} \boldsymbol{n}=\left(n^{x}, n^{y}, n^{z}\right)^{\mathrm{T}}$ are the coordinates of the outward unit normal $\boldsymbol{n}$ of $\Omega$.

In order to evaluate the principle of virtual work (5), the total virtual work $\delta W_{\text {tot }}$ of the body is required. The total virtual work is constituted of the virtual work contribution of the internal forces $\delta W^{\mathrm{i}}$ and the external forces $\delta W^{\mathrm{e}}$. Hence, the principle of virtual work (5) takes the form

$$
\delta W_{\text {tot }}=\delta W^{\mathrm{i}}+\delta W^{\mathrm{e}}=0 \quad \text { for all } \delta \xi .
$$


Since only stresses are allowed as internal forces, the internal forces contribute according to (44) as

$$
\delta W^{\mathrm{i}}=\delta W^{\mathrm{s}}=-\int_{\Omega} \frac{\partial \delta \boldsymbol{\xi}}{\partial \boldsymbol{x}}: \boldsymbol{\sigma} \mathrm{d} v=-\int_{\Omega}\left(\delta \boldsymbol{\xi}_{, x} \cdot \boldsymbol{t}_{x}+\delta \boldsymbol{\xi}_{, y} \cdot \boldsymbol{t}_{y}+\delta \boldsymbol{\xi}_{, z} \cdot \boldsymbol{t}_{z}\right) \mathrm{d} v .
$$

To avoid the divergence theorem for (second-order) tensors, we will use partial integration for the virtual work contribution $\delta W^{\mathrm{i}}$ in terms of the cartesian stress vectors $t_{i}$, as shown in the right part of (64). The application of this procedure to curvilinear coordinates is presented in the Appendix for the example of cylindrical coordinates. For now using the product rule, each of the three terms $\delta \boldsymbol{\xi}_{, i} \cdot \boldsymbol{t}_{i}$ in (64) is rewritten as

$$
\delta \boldsymbol{\xi}_{, x} \cdot \boldsymbol{t}_{x}=\left[\delta \boldsymbol{\xi} \cdot \boldsymbol{t}_{x}\right]_{, x}-\delta \boldsymbol{\xi} \cdot \boldsymbol{t}_{x, x} \quad(\text { same for } y \text { and } z),
$$

with which (64) becomes

$$
\delta W^{\mathrm{i}}=-\int_{\Omega}\left(\left[\delta \boldsymbol{\xi} \cdot \boldsymbol{t}_{x}\right]_{, x}+\left[\delta \boldsymbol{\xi} \cdot \boldsymbol{t}_{y}\right]_{, y}+\left[\delta \boldsymbol{\xi} \cdot \boldsymbol{t}_{z}\right]_{, z}\right) \mathrm{d} v+\int_{\Omega} \delta \boldsymbol{\xi} \cdot\left(\boldsymbol{t}_{x, x}+\boldsymbol{t}_{y, y}+\boldsymbol{t}_{z, z}\right) \mathrm{d} v .
$$

Applying now the divergence theorem (62) on each of the three terms $\left[\delta \boldsymbol{\xi} \cdot \boldsymbol{t}_{i}\right]_{, i}$ in the first integral, we obtain

$$
\begin{aligned}
\delta W^{\mathrm{i}}=-\int_{\partial \Omega}\left(\left[\delta \boldsymbol{\xi} \cdot \boldsymbol{t}_{x}\right] n^{x}+\left[\delta \boldsymbol{\xi} \cdot \boldsymbol{t}_{y}\right] n^{y}+\left[\delta \boldsymbol{\xi} \cdot \boldsymbol{t}_{z}\right] n^{z}\right) \mathrm{d} a & \\
& +\int_{\Omega} \delta \boldsymbol{\xi} \cdot\left(\boldsymbol{t}_{x, x}+\boldsymbol{t}_{y, y}+\boldsymbol{t}_{z, z}\right) \mathrm{d} v .
\end{aligned}
$$

With $\boldsymbol{n}=n^{x} \boldsymbol{e}_{x}^{I}+n^{y} \boldsymbol{e}_{y}^{I}+n^{z} \boldsymbol{e}_{z}^{I}$ and the definition of the stress tensor $\boldsymbol{\sigma}$ according to (28), the virtual work contribution (67) may now be rewritten as

$$
\delta W^{\mathrm{i}}=-\int_{\partial \Omega} \delta \boldsymbol{\xi} \cdot \boldsymbol{\sigma} \cdot \boldsymbol{n} \mathrm{d} a+\int_{\Omega} \delta \boldsymbol{\xi} \cdot\left(\boldsymbol{t}_{x, x}+\boldsymbol{t}_{y, y}+\boldsymbol{t}_{z, z}\right) \mathrm{d} v,
$$

which is the desired strong variational form. By following our assumption about the external loading of the body, only volume forces $f_{v}$ and surface forces $f_{a}$ are considered. The external virtual work is therefore given by (11) as

$$
\delta W^{\mathrm{e}}=\int_{\Omega} \delta \boldsymbol{\xi} \cdot \boldsymbol{f}_{v} \mathrm{~d} v+\int_{\partial \Omega} \delta \boldsymbol{\xi} \cdot \boldsymbol{f}_{a} \mathrm{~d} a .
$$

The total virtual work (63) is obtained by summing up (68) and (69), which gives the strong variational form of the principle of virtual work in the deformed configuration $\bar{\Omega}$ :

$\delta W_{\mathrm{tot}}=\int_{\Omega} \delta \boldsymbol{\xi} \cdot\left(\boldsymbol{t}_{x, x}+\boldsymbol{t}_{y, y}+\boldsymbol{t}_{z, z}+\boldsymbol{f}_{v}\right) \mathrm{d} v+\int_{\partial \Omega} \delta \boldsymbol{\xi} \cdot\left(\boldsymbol{f}_{a}-\boldsymbol{\sigma} \cdot \boldsymbol{n}\right) \mathrm{d} a=0$ 
We now apply again the fundamental lemma of calculus of variations under the following choices of variations: in a first step, we choose virtual displacement fields with $\delta \xi=0$ on the boundary $\partial \Omega$ and $\delta \xi$ arbitrary in the interior $\Omega$, and in a second step, we let $\delta \xi$ be arbitrary on the boundary $\partial \Omega$. This results in the two localized conditions

$$
\begin{aligned}
& \boldsymbol{t}_{x, x}+\boldsymbol{t}_{y, y}+\boldsymbol{t}_{z, z}=-\boldsymbol{f}_{v} \quad \text { in } \Omega \quad \text { (local equilibrium conditions), } \\
& \boldsymbol{\sigma} \cdot \boldsymbol{n}=\boldsymbol{f}_{a} \quad \text { on } \partial \Omega \quad \text { (boundary conditions), }
\end{aligned}
$$

which are the partial differential equations in the interior $\Omega$ and the boundary conditions for the forces on $\partial \Omega$, forming together the classical boundary value problem of continuum mechanics.

\section{Integral balance laws}

So far, we have not yet drawn any conclusions about how the subsystems of $\bar{\Omega}$ interact with each other. The answer to this question will be given at the end of this section in the inverse stress theorem, of which the argumentation follows the opposite direction as in Cauchy's stress theorem. The interaction between the subsystems can only take place by their external forces, since their internal forces, by definition, cannot interact with their environments. Hence, we need to determine the external forces for an arbitrary subsystem $\bar{K}$ of the continuum. For this, the total virtual work of $\bar{\Omega}$ is required and has to be evaluated for those virtual displacement fields that correspond with rigid virtual displacements on the considered subsystem $\bar{K}$ and are zero elsewhere. By doing so, the internal forces of $\bar{K}$ drop out, as do any other forces not acting on the points of $\bar{K}$, and only the external forces of $\bar{K}$ remain. This special choice of virtual displacement fields is neither smooth nor continuous. But as has already been recognized by [Hellinger 1914, §3.d], which is commented on in [Eugster and dell'Isola 2017a], one succeeds with approximating such fields by a family of continuous virtual displacements. Strichartz [1994, §6.6] shows that such an approximation can even be achieved with smooth test functions, i.e., with smooth virtual displacement fields.

For the sake of brevity and without loss of generality, we choose $\bar{K}$ to be an inner subsystem. An inner subsystem $\bar{K}$ of $\bar{\Omega}$ is understood in this context to be a closed subset of $\bar{\Omega}$, whose boundary $\partial K$ does not have any material points in common with the boundary $\partial \Omega$ of $\bar{\Omega}$. As depicted in Figure 5 , we denote the boundary of $\bar{K}$ by $\partial K$, the interior of $\bar{K}$ by $K$, and the complement of $\bar{K}$ with respect to $\bar{\Omega}$ as $H:=\bar{\Omega} \backslash \bar{K}$. Hence, $\bar{\Omega}$ can be subdivided into the disjoint sets

$$
\bar{\Omega}=K \cup \partial K \cup H=\bar{K} \cup H \quad \text { with } \bar{K}=K \cup \partial K \text {. }
$$



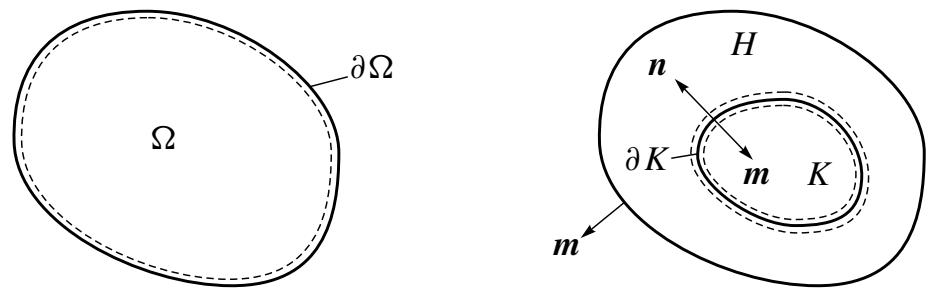

Figure 5. Disjoint subdivision of the deformed configuration $\bar{\Omega}$ into an inner subsystem $\bar{K}$ and its complement $H=\bar{\Omega} \backslash \bar{K}$. The outward normals of $K$ and $H$ are denoted by $\boldsymbol{n}$ and $\boldsymbol{m}$, respectively. The common boundary of $K$ and $H$ is given by the boundary of $K$, denoted as $\partial K$.

For the boundaries, we have

$$
\partial H=\partial \Omega \cup \partial K \quad \text { with } \boldsymbol{m}=-\boldsymbol{n} \text { on } \partial K,
$$

where $\boldsymbol{n}$ and $\boldsymbol{m}$ are the outward-pointing unit normals of $K$ and $H$, respectively.

For the formulation of the total virtual work $\delta W_{\text {tot }}$ of $\bar{\Omega}$ according to (70), we sum up the contributions of the total virtual work $\delta W_{\text {tot }}^{H}$ and $\delta W_{\text {tot }}^{K}$ of $H$ and $K$, respectively. For $\delta W_{\text {tot }}^{H}$, we obtain similarly to (70)

$$
\begin{array}{r}
\delta W_{\mathrm{tot}}^{H}=\int_{H} \delta \boldsymbol{\xi} \cdot\left(\boldsymbol{t}_{x, x}+\boldsymbol{t}_{y, y}+\boldsymbol{t}_{z, z}+\boldsymbol{f}_{v}\right) \mathrm{d} v+\int_{\partial \Omega} \delta \boldsymbol{\xi} \cdot\left(\boldsymbol{f}_{a}-\boldsymbol{\sigma} \cdot \boldsymbol{m}\right) \mathrm{d} a \\
-\int_{\partial K} \delta \boldsymbol{\xi} \cdot \boldsymbol{\sigma} \cdot \boldsymbol{m} \mathrm{d} a,
\end{array}
$$

where, according to our loading assumptions, the external surface forces $f_{a}$ of $\bar{\Omega}$ act only on the subset $\partial \Omega$ of $\partial H$, but not on the boundary $\partial K$ that was generated by the subdivision (72) and (73). Consequently, the total virtual work $\delta W_{\text {tot }}^{K}$ of $K$ becomes

$$
\delta W_{\mathrm{tot}}^{K}=\int_{K} \delta \boldsymbol{\xi} \cdot\left(\boldsymbol{t}_{x, x}+\boldsymbol{t}_{y, y}+\boldsymbol{t}_{z, z}+\boldsymbol{f}_{v}\right) \mathrm{d} v-\int_{\partial K} \delta \boldsymbol{\xi} \cdot \boldsymbol{\sigma} \cdot \boldsymbol{n} \mathrm{d} a .
$$

By adding (74) and (75), one obtains

$$
\delta W_{\mathrm{tot}}^{H}+\delta W_{\mathrm{tot}}^{K}=\delta W_{\mathrm{tot}},
$$

which is, due to $\boldsymbol{m}=-\boldsymbol{n}$ on $\partial K$, again the virtual work according to (70).

To extract now from the total virtual work (76) only the external virtual work contribution of $\bar{K}$, we choose for $\delta \xi$ the rigid virtual displacements on $\bar{K}$, which implies

$$
\delta W_{\text {tot }}=0 \quad \text { for all } \delta \boldsymbol{\xi}_{\bar{K}, \text { rig }}(\boldsymbol{x})= \begin{cases}\delta \boldsymbol{\xi}_{\text {rig }}(\boldsymbol{x}) & \text { for } \boldsymbol{x} \in \bar{K}=K \cup \partial K, \\ 0 & \text { for } \boldsymbol{x} \in H .\end{cases}
$$


With $\delta \boldsymbol{\xi}_{\bar{K}, \text { rig }}(\boldsymbol{x})=0$ for $\boldsymbol{x} \in H$, the virtual work contributions

$$
\begin{array}{r}
\int_{H} \delta \boldsymbol{\xi}_{\bar{K}, \text { rig }} \cdot\left(\boldsymbol{t}_{x, x}+\boldsymbol{t}_{y, y}+\boldsymbol{t}_{z, z}+\boldsymbol{f}_{v}\right) \mathrm{d} v=0, \\
\int_{\partial \Omega} \delta \boldsymbol{\xi}_{\bar{K}, \text { rig }} \cdot\left(\boldsymbol{f}_{a}-\boldsymbol{\sigma} \cdot \boldsymbol{m}\right) \mathrm{d} a=0
\end{array}
$$

vanish from $\delta W_{\text {tot }}^{H}$ in (74), and the variational problem (76) and (77) reduces with the remaining contributions from (74) and (75) to

$\delta W_{\mathrm{tot}}=\int_{K} \delta \boldsymbol{\xi}_{\mathrm{rig}} \cdot\left(\boldsymbol{t}_{x, x}+\boldsymbol{t}_{y, y}+\boldsymbol{t}_{z, z}+\boldsymbol{f}_{v}\right) \mathrm{d} v-\int_{\partial K} \delta \boldsymbol{\xi}_{\mathrm{rig}} \cdot(\boldsymbol{\sigma} \cdot \boldsymbol{n}+\boldsymbol{\sigma} \cdot \boldsymbol{m}) \mathrm{d} a=0$
for all $\delta \boldsymbol{\xi}_{\mathrm{rig}}$.

The contribution of the internal forces of $K$, consisting in our case of just the stresses in $K$, cancels out of (79) because of (41). To demonstrate this explicitly, we reverse the partial integration (64)-(68), which gives

$$
\int_{K} \delta \boldsymbol{\xi}_{\mathrm{rig}} \cdot\left(\boldsymbol{t}_{x, x}+\boldsymbol{t}_{y, y}+\boldsymbol{t}_{z, z}\right) \mathrm{d} v-\int_{\partial K} \delta \boldsymbol{\xi}_{\mathrm{rig}} \cdot \boldsymbol{\sigma} \cdot \boldsymbol{n} \mathrm{d} a=-\int_{K} \frac{\partial \delta \boldsymbol{\xi}_{\mathrm{rig}}}{\partial \boldsymbol{x}}: \boldsymbol{\sigma} \mathrm{d} v=0
$$

According to this, (79) reduces to

$$
\delta W_{\mathrm{tot}}=\int_{K} \delta \boldsymbol{\xi}_{\mathrm{rig}} \cdot \boldsymbol{f}_{v} \mathrm{~d} v-\int_{\partial K} \delta \boldsymbol{\xi}_{\mathrm{rig}} \cdot \boldsymbol{\sigma} \cdot \boldsymbol{m} \mathrm{d} a=0 \quad \text { for all } \delta \boldsymbol{\xi}_{\mathrm{rig}} .
$$

In addition, the identity $\boldsymbol{m}=-\boldsymbol{n}$ holds on $\partial K$ by (73) for the outward normal $\boldsymbol{m}$ of $H$. Furthermore, by setting

$$
t:=\sigma \cdot n
$$

we can reformulate (81) as

$$
0=\int_{K} \delta \xi_{\text {rig }} \cdot \boldsymbol{f}_{v} \mathrm{~d} v+\int_{\partial K} \delta \boldsymbol{\xi}_{\mathrm{rig}} \cdot \boldsymbol{t} \mathrm{d} a \quad \text { for all } \delta \boldsymbol{\xi}_{\text {rig }} .
$$

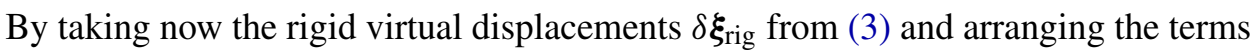
with respect to $\delta \boldsymbol{r}_{O}$ and $\delta \boldsymbol{\phi}$, one obtains

$$
\begin{aligned}
& 0=\int_{K}\left(\delta \boldsymbol{r}_{O}+\delta \boldsymbol{\phi} \times \boldsymbol{x}\right) \cdot \boldsymbol{f}_{v} \mathrm{~d} v+\int_{\partial K}\left(\delta \boldsymbol{r}_{O}+\delta \boldsymbol{\phi} \times \boldsymbol{x}\right) \cdot \boldsymbol{t} \mathrm{d} a \\
&=\delta \boldsymbol{r}_{O} \cdot\left(\int_{K} \boldsymbol{f}_{v} \mathrm{~d} v+\int_{\partial K} \boldsymbol{t} \mathrm{d} a\right)+\delta \boldsymbol{\phi} \cdot\left(\int_{K} \boldsymbol{x} \times \boldsymbol{f}_{V} \mathrm{~d} v+\int_{\partial K} \boldsymbol{x} \times \boldsymbol{t} \mathrm{d} a\right) \\
& \text { for all } \delta \boldsymbol{r}_{O} \text { and all } \delta \boldsymbol{\phi} .
\end{aligned}
$$

After the evaluation of the two independent variations for $\delta \boldsymbol{r}_{O}$ and $\delta \boldsymbol{\phi}$, one ends up 
with the integral balance laws for the external forces and moments acting on $\bar{K}$ :

$$
\begin{aligned}
\int_{K} \boldsymbol{f}_{v} \mathrm{~d} v+\int_{\partial K} \boldsymbol{t} \mathrm{d} a & =0 \quad \text { (equilibrium of external forces), } \\
\int_{K} \boldsymbol{x} \times \boldsymbol{f}_{v} \mathrm{~d} v+\int_{\partial K} \boldsymbol{x} \times \boldsymbol{t} \mathrm{d} a & =0 \quad \text { (equilibrium of external moments). }
\end{aligned}
$$

With $\boldsymbol{t}=\boldsymbol{\sigma} \cdot \boldsymbol{n}$ from (82) and $\boldsymbol{m}=-\boldsymbol{n}$ from (73), it is now also apparent, how the subsystems of $\bar{\Omega}$ interact with each other:

Theorem 1 (inverse stress theorem). Let the internal forces of $\bar{\Omega}$ consist only of the continuous stress tensor field $\sigma$, and let $\bar{K}$ be an (inner) subsystem of $\bar{\Omega}$. Then the complement $H=\bar{\Omega} \backslash \bar{K}$ of $\bar{K}$ exerts on the (inner) subsystem $\bar{K}$ the force and the moment

$$
\int_{\partial K} \boldsymbol{t} \mathrm{d} a \text { and } \int_{\partial K} \boldsymbol{x} \times \boldsymbol{t} \mathrm{d} a
$$

via the surface $\partial K$ of $\bar{K}$, where $t$ depends linearly on the outward-pointing unit normal $\boldsymbol{n}$ of $\bar{K}$ by $\boldsymbol{t}=\boldsymbol{\sigma} \cdot \boldsymbol{n}$.

In the nonvariational approach to mechanics, the stress principle of Euler and Cauchy assumes the interaction between subsystems to take place by surface forces only. Cauchy's stress theorem then shows that these surface forces depend linearly on the normal of the contact surface, which asserts the existence of the stress tensor field. Since we proposed in this paper an alternative notion of stress, the stress tensor field follows already from the derivation in Section 4, but the interaction mechanism between the subsystems remains unexplained. This interaction mechanism is now formulated in Theorem 1, called the inverse stress theorem, as it demonstrates the reverse direction of Cauchy's stress theorem.

\section{Conclusion}

In this paper, the controversy about the a priori notion of the contribution of the internal virtual work in a variational formulation has been resolved by an alternative perspective on the notion of stress. This notion relies on the interpretation of Piola's micro-macro identification procedure in view of the Riemann integral, which naturally provides in its mathematical definition a micro-macro relation between the discrete system of infinitesimal volume elements and the continuum. Accordingly, we proposed a definition of stress in the sense of Boltzmann on the micro level of the infinitesimal volume elements. In particular, when the stress is defined as the internal force effects of the body that model the mutual force interaction between neighboring infinitesimal volume elements, Piola's micro-macro identification procedure leads directly to the internal virtual work of the classical continuum. In the course of this, the stress tensor emerges as the quantity dual to 
the gradient of the virtual displacement field. It seems that Piola's micro-macro identification procedure gives us a very intuitive tool to model a wide variety of force interactions which can appear in a continuum.

In contrast to the nonvariational formulation, in which the stress principle of Euler and Cauchy defines the concept of stress, no assumption has been made about the mechanism of interaction between subsystems, in order to show the existence of the stress tensor. Within the variational formulation, the mechanism of interaction between the subsystems follows from the inverse stress theorem, which effectively shows the reverse direction of Cauchy's stress theorem. The derivation of the virtual work contribution of the stress as given in Section 4, together with the inverse stress theorem, can be considered as the variational counterpart to Cauchy's stress theorem. In the variational formulation, the symmetry of the stress tensor is a direct consequence of the variational law of interaction and the continuity of the stress field. Since the virtual work is by definition an invariant expression, the virtual work provides for different integral parametrizations or coordinate representations the required transformation properties.

As variational methods are inevitable for generalized continua, i.e., continua with microstructure or higher-order continua, it needs to be shown how the introduced notion of stress can be applied to those theories. In the present approach, neighboring volume elements have been understood as adjacent volume elements sharing the same surface. However, Piola's nonlocal theory makes us confident to conjecture that the consideration of additional interactions between volume elements sharing the same edges and wedges, or which are even nonadjacent, will lead to higher-order stresses as known from $N$-th gradient theories. Nevertheless, the proposed definition of stress on the micro level of infinitesimal volume elements would still hold in its essence.

In order to focus on the concept of stress, we have omitted the discussion about inertia forces and inertial frames. By adding the inertia forces in the sense of d'Alembert as external volume forces to the total virtual work, the equations of motion in variational form and consequently a theory of dynamics is obtained. Such a variational formulation already forms the basis for many approaches in analytical dynamics, as for example the Lagrangian equations of motion, Hamilton's principle, or the projected Newton-Euler equations. As a consequence, both continuum mechanics and analytical dynamics are based on the very same axioms. The theories differ merely in the modeling of the corresponding force interactions.

\section{Appendix: Equilibrium equations in cylindrical coordinates}

In order to avoid the concept of co- and contravariant basis vectors, (50) is a convenient starting point to derive the local equilibrium conditions also for curvilinear 
coordinates. As an example, we choose cylindrical coordinates $(p, q, r)=(r, \varphi, z)$ which are related to the cartesian coordinates by

$$
{ }_{I} \boldsymbol{x}=\boldsymbol{\varphi}(r, \varphi, z)=(r \cos \varphi, r \sin \varphi, z)^{\mathrm{T}},
$$

with the determinant of the Jacobian $J=\operatorname{det}(D \varphi)(r, \varphi, z)=r$. The virtual work of internal forces according to (50) is

$$
\delta W^{\mathrm{i}}=-\int_{\Omega}\left(\delta \boldsymbol{\xi}_{, r} \cdot \boldsymbol{t}_{r}+\delta \boldsymbol{\xi}_{, \varphi} \cdot \boldsymbol{t}_{\varphi}+\delta \boldsymbol{\xi}_{, z} \cdot \boldsymbol{t}_{z}\right) r \mathrm{~d} v,
$$

where we have omitted the hat and have used the volume element (46). With the volume element $r \mathrm{~d} v=r \mathrm{~d} r \mathrm{~d} \varphi \mathrm{d} z$, the virtual work densities within the integral have the units $\left[\delta \boldsymbol{\xi}_{, i} \cdot \boldsymbol{t}_{i}\right]=\mathrm{Nm} / \mathrm{m}^{3}$. In contrast to $\boldsymbol{t}_{r}$ and $\boldsymbol{t}_{z}$ having units $\left[\boldsymbol{t}_{i}\right]=\mathrm{N} / \mathrm{m}^{2}$, the stress vector $\boldsymbol{t}_{\varphi}$ has, due to the dimensionless partial derivative $\delta \boldsymbol{\xi}_{, \varphi}$, the unit $\left[\boldsymbol{t}_{\varphi}\right]=\mathrm{N} / \mathrm{m}^{3}$. This dimensional mismatch can be corrected by introducing the socalled physical stress vectors $\boldsymbol{\sigma}_{r}, \boldsymbol{\sigma}_{\varphi}, \boldsymbol{\sigma}_{z}$ [Başar and Weichert 2000, p. 101], defined by the relations

$$
\boldsymbol{t}_{r}=\boldsymbol{\sigma}_{r}, \quad \boldsymbol{t}_{\varphi}=\frac{1}{r} \boldsymbol{\sigma}_{\varphi}, \quad \boldsymbol{t}_{z}=\boldsymbol{\sigma}_{z} .
$$

The virtual work (88) together with the physical stress vectors (89) leads to ${ }^{16}$

$$
\delta W^{\mathrm{i}}=-\int_{\Omega}\left(\delta \boldsymbol{\xi}_{, r} \cdot \boldsymbol{\sigma}_{r}+\delta \boldsymbol{\xi}_{, \varphi} \cdot \frac{1}{r} \boldsymbol{\sigma}_{\varphi}+\delta \boldsymbol{\xi}_{, z} \cdot \boldsymbol{\sigma}_{z}\right) r \mathrm{~d} v .
$$

By using the product rule, we can reformulate each of the terms in (90) according to

$$
\begin{aligned}
\delta \boldsymbol{\xi}_{, r} \cdot \boldsymbol{\sigma}_{r} r & =\left[\delta \boldsymbol{\xi} \cdot \boldsymbol{\sigma}_{r} r\right]_{, r}-\delta \boldsymbol{\xi} \cdot\left[\boldsymbol{\sigma}_{r, r}+\frac{1}{r} \boldsymbol{\sigma}_{r}\right] r, \\
\delta \boldsymbol{\xi}_{, \varphi} \cdot \boldsymbol{\sigma}_{\varphi} & =\left[\delta \boldsymbol{\xi} \cdot \boldsymbol{\sigma}_{\varphi}\right]_{, \varphi}-\delta \boldsymbol{\xi} \cdot\left[\frac{1}{r} \boldsymbol{\sigma}_{\varphi, \varphi}\right] r, \\
\delta \boldsymbol{\xi}_{, z} \cdot \boldsymbol{\sigma}_{z} r & =\left[\delta \boldsymbol{\xi} \cdot \boldsymbol{\sigma}_{z} r\right]_{, z}-\delta \boldsymbol{\xi} \cdot \boldsymbol{\sigma}_{z, z} r .
\end{aligned}
$$

Applying the identities (91) to (88), we reformulate the internal virtual work as

$$
\begin{aligned}
\delta W^{\mathrm{i}}=-\int_{\Omega}\left(\left[\delta \boldsymbol{\xi} \cdot \boldsymbol{\sigma}_{r} r\right]_{, r}+\right. & {\left.\left[\delta \boldsymbol{\xi} \cdot \boldsymbol{\sigma}_{\varphi}\right]_{, \varphi}+\left[\delta \boldsymbol{\xi} \cdot \boldsymbol{\sigma}_{z} r\right]_{, z}\right) \mathrm{d} r \mathrm{~d} \varphi \mathrm{d} z } \\
& +\int_{\Omega} \delta \boldsymbol{\xi} \cdot\left(\boldsymbol{\sigma}_{r, r}+\frac{1}{r} \boldsymbol{\sigma}_{r}+\frac{1}{r} \boldsymbol{\sigma}_{\varphi, \varphi}+\boldsymbol{\sigma}_{z, z}\right) r \mathrm{~d} r \mathrm{~d} \varphi \mathrm{d} z .
\end{aligned}
$$

After carrying out the integration (62) of the first term in (92), the virtual displacements are chosen such that $\delta \xi=0$ on the boundary $\partial \Omega$, which yields

$$
\delta W^{\mathrm{i}}=\int_{\Omega} \delta \xi \cdot\left(\sigma_{r, r}+\frac{1}{r} \sigma_{r}+\frac{1}{r} \sigma_{\varphi, \varphi}+\sigma_{z, z}\right) r \mathrm{~d} r \mathrm{~d} \varphi \mathrm{d} z .
$$

${ }^{16}$ Alternatively, (90) can be obtained by evaluating the expression $\partial \delta \boldsymbol{\xi} / \partial \boldsymbol{x}: \boldsymbol{\sigma}=\left(\delta \boldsymbol{\xi}_{, r} \otimes \partial r / \partial \boldsymbol{x}+\right.$ $\left.\delta \boldsymbol{\xi}_{, \varphi} \otimes \partial \varphi / \partial \boldsymbol{x}+\delta \boldsymbol{\xi}_{, z} \otimes \partial z / \partial \boldsymbol{x}\right):\left(\boldsymbol{\sigma}_{r} \otimes \boldsymbol{e}_{r}+\boldsymbol{\sigma}_{\varphi} \otimes \boldsymbol{e}_{\varphi}+\boldsymbol{\sigma}_{z} \otimes \boldsymbol{e}_{z}\right)$ using the basis vectors of (97). 
As in Section 8, the external forces are assumed to be either volume forces or surface forces on the boundary of the body. Consequently, just the volume forces $\boldsymbol{f}_{v}$ contribute to the virtual work,

$$
\delta W^{\mathrm{e}}=\int_{\Omega} \delta \xi \cdot f_{v} r \mathrm{~d} r \mathrm{~d} \varphi \mathrm{d} z
$$

as the virtual displacements have been chosen to vanish on the boundary. With $\delta W_{\text {tot }}=\delta W^{\mathrm{i}}+\delta W^{\mathrm{e}}$ together with the contributions (93) and (94), the principle of virtual work (5) implies that

$$
\delta W_{\mathrm{tot}}=\int_{\Omega} \delta \xi \cdot\left(\boldsymbol{\sigma}_{r, r}+\frac{1}{r} \boldsymbol{\sigma}_{r}+\frac{1}{r} \boldsymbol{\sigma}_{\varphi, \varphi}+\boldsymbol{\sigma}_{z, z}+\boldsymbol{f}_{v}\right) r \mathrm{~d} r \mathrm{~d} \varphi \mathrm{d} z=0 \quad \text { for all } \delta \xi
$$

By the fundamental lemma of calculus of variations, the strong variational form (95) leads directly to the local equilibrium conditions

$$
\boldsymbol{\sigma}_{r, r}+\frac{1}{r} \sigma_{r}+\frac{1}{r} \sigma_{\varphi, \varphi}+\sigma_{z, z}+f_{v}=0
$$

It is convenient to represent the physical stress vectors in the cylindrical orthonormal basis $\left(\boldsymbol{e}_{r}, \boldsymbol{e}_{\varphi}, \boldsymbol{e}_{z}\right)$, which depends on its position $(r, \varphi, z)$ and is defined with respect to the $I$-system as

$$
{ }_{I} \boldsymbol{e}_{r}=\left(\begin{array}{c}
\cos \varphi \\
\sin \varphi \\
0
\end{array}\right), \quad{ }_{I} \boldsymbol{e}_{\varphi}=\left(\begin{array}{c}
-\sin \varphi \\
\cos \varphi \\
0
\end{array}\right), \quad{ }_{I} \boldsymbol{e}_{z}=\left(\begin{array}{l}
0 \\
0 \\
1
\end{array}\right)
$$

By computing the partial derivative of the basis vectors with respect to $(r, \varphi, z)$, it can easily be shown that the only nonvanishing contributions are

$$
\boldsymbol{e}_{r, \varphi}=\boldsymbol{e}_{\varphi}, \quad \boldsymbol{e}_{\varphi, \varphi}=-\boldsymbol{e}_{r}
$$

For an explicit evaluation of (96) in components, the following computations are of importance:

$$
\begin{aligned}
& \boldsymbol{\sigma}_{r, r}=\left(\sigma_{r r} \boldsymbol{e}_{r}+\tau_{\varphi r} \boldsymbol{e}_{\varphi}+\tau_{z r} \boldsymbol{e}_{z}\right)_{, r}=\left(\sigma_{r r, r} \boldsymbol{e}_{r}+\tau_{\varphi r, r} \boldsymbol{e}_{\varphi}+\tau_{z r, r} \boldsymbol{e}_{z}\right), \\
& \boldsymbol{\sigma}_{\varphi, \varphi}=\left(\tau_{r \varphi} \boldsymbol{e}_{r}+\sigma_{\varphi \varphi} \boldsymbol{e}_{\varphi}+\tau_{z \varphi} \boldsymbol{e}_{z}\right)_{, \varphi}=\left(\tau_{r \varphi, \varphi} \boldsymbol{e}_{r}+\tau_{r \varphi} \boldsymbol{e}_{\varphi}+\sigma_{\varphi \varphi, \varphi} \boldsymbol{e}_{\varphi}-\sigma_{\varphi \varphi} \boldsymbol{e}_{r}+\tau_{z \varphi, \varphi} \boldsymbol{e}_{z}\right), \\
& \boldsymbol{\sigma}_{z, z}=\left(\tau_{r z} \boldsymbol{e}_{r}+\tau_{\varphi z} \boldsymbol{e}_{\varphi}+\boldsymbol{\sigma}_{z z} \boldsymbol{e}_{z}\right)_{, z}=\tau_{r z, r} \boldsymbol{e}_{r}+\tau_{\varphi z, z} \boldsymbol{e}_{\varphi}+\sigma_{z z, z} \boldsymbol{e}_{z}, \\
& \boldsymbol{f}_{v}=f_{r} \boldsymbol{e}_{r}+f_{\varphi} \boldsymbol{e}_{\varphi}+f_{z} \boldsymbol{e}_{z} .
\end{aligned}
$$


Using (99) within (96), we end up with the local equilibrium equations described in the components of the $\left(\boldsymbol{e}_{r}, \boldsymbol{e}_{\varphi}, \boldsymbol{e}_{z}\right)$-system:

$$
\begin{aligned}
& 0=\sigma_{r r, r}+\frac{1}{r} \sigma_{r r}+\frac{1}{r} \tau_{r \varphi, \varphi}-\frac{1}{r} \sigma_{\varphi \varphi}+\tau_{r z, z}+f_{r}, \\
& 0=\tau_{\varphi r, r}+\frac{1}{r} \tau_{\varphi r}+\frac{1}{r} \sigma_{\varphi \varphi, \varphi}+\frac{1}{r} \tau_{r \varphi}+\tau_{\varphi z, z}+f_{\varphi}, \\
& 0=\tau_{z r, r}+\frac{1}{r} \tau_{z r}+\frac{1}{r} \tau_{z \varphi, \varphi}+\sigma_{z z, z}+f_{z},
\end{aligned}
$$

which is often derived by geometrical arguments on the cylindrical volume elements [Timoshenko and Goodier 1951, p. 55]. The same procedure as proposed here can also be applied to obtain the Lagrangian equilibrium equation in cylindrical and spherical coordinates, which are presented in [Volokh 2006].

\section{References}

[Altenbach and Altenbach 1994] J. Altenbach and H. Altenbach, Einführung in die Kontinuumsmechanik, Teubner, 1994.

[Başar and Weichert 2000] Y. Başar and D. Weichert, Nonlinear continuum mechanics of solids: fundamental mathematical and physical concepts, Springer, 2000.

[Becker and Bürger 1975] E. Becker and W. Bürger, Kontinuumsmechanik: eine Einführung in die Grundlagen und einfache Anwendungen, Leitfäden der Angewandten Mathematik und Mechanik 20, Teubner, 1975.

[Bertram 1989] A. Bertram, Axiomatische Einführung in die Kontinuumsmechanik, Bibliographisches Institut, 1989.

[Bertram 2012] A. Bertram, Elasticity and plasticity of large deformations: an introduction, 3rd ed., Springer, 2012.

[Bertram and Forest 2007] A. Bertram and S. Forest, "Mechanics based on an objective power functional", Tech. Mech. 27:1 (2007), 1-17.

[Boltzmann 1905] L. Boltzmann, Populäre Schriften, Barth, 1905.

[Bonet and Wood 1997] J. Bonet and R. D. Wood, Nonlinear continuum mechanics for finite element analysis, Cambridge University, 1997.

[Capecchi and Ruta 2015] D. Capecchi and G. Ruta, Strength of materials and theory of elasticity in 19th century Italy: a brief account of the history of mechanics of solids and structures, Advanced Structured Materials 52, Springer, 2015.

[Cauchy 1823] A. L. Cauchy, "Recherches sur l'équilibre et le mouvement intérieur des corps solides ou fluides, élastiques ou non élastiques", pp. 300-304 in Euvres complètes, II, tome II, Gauthier-Villars, 1823.

[Cauchy 1827a] A. L. Cauchy, "De la pression ou tension dans un corps solide", pp. 60-78 in CEuvres complètes, II, tome VII, Gauthier-Villars, 1827.

[Cauchy 1827b] A. L. Cauchy, "Sur les relations qui existent dans l'état d'équilibre d'un corps solide ou fluide, entre les pressions ou tensions et les forces accélératrices", pp. 141-145 in Euvres complètes, II, tome VII, Gauthier-Villars, 1827.

[Chadwick 1999] P. Chadwick, Continuum mechanics: concise theory and problems, 2nd ed., Dover, 1999. 
[Ciarlet 1988] P. G. Ciarlet, Mathematical elasticity, vol. I: Three-dimensional elasticity, Studies in Mathematics and its Applications 20, North-Holland, 1988.

[Clebsch 1862] A. Clebsch, Theorie der Elasticität fester Körper, Teubner, 1862.

[Del Piero 2009] G. Del Piero, "On the method of virtual power in continuum mechanics", J. Mech. Mater. Struct. 4:2 (2009), 281-292.

[dell'Isola et al. 2011] F. dell'Isola, P. Seppecher, and A. Madeo, "Beyond Euler-Cauchy continua: the structure of contact actions in $N$-th gradient generalized continua: a generalization of the Cauchy tetrahedron argument", pp. 17-106 in Variational models and methods in solid and fluid mechanics, edited by F. dell'Isola and S. Gavrilyuk, CISM Courses and Lectures 535, Springer, 2011.

[dell'Isola et al. 2012] F. dell'Isola, P. Seppecher, and A. Madeo, "How contact interactions may depend on the shape of Cauchy cuts in $N$ th gradient continua: approach 'à la D'Alembert"', $Z$. Angew. Math. Phys. 63:6 (2012), 1119-1141.

[dell'Isola et al. 2015a] F. dell'Isola, U. Andreaus, and L. Placidi, "At the origins and in the vanguard of peridynamics, non-local and higher-gradient continuum mechanics: an underestimated and still topical contribution of Gabrio Piola", Math. Mech. Solids 20:8 (2015), 887-928.

[dell'Isola et al. 2015b] F. dell'Isola, P. Seppecher, and A. Della Corte, "The postulations á la D'Alembert and á la Cauchy for higher gradient continuum theories are equivalent: a review of existing results", Proc. A. 471:2183 (2015), 20150415.

[dell' Isola et al. 2017] F. dell'Isola, A. Della Corte, and I. Giorgio, "Higher-gradient continua: the legacy of Piola, Mindlin, Sedov and Toupin and some future research perspectives", Math. Mech. Solids 22:4 (2017), 852-872.

[Dvorkin and Goldschmit 2006] E. N. Dvorkin and M. B. Goldschmit, Nonlinear continua, Springer, 2006.

[Eringen 1980] A. C. Eringen, Mechanics of continua, 2nd ed., Krieger, 1980.

[Eugster 2015a] S. Eugster, Geometric continuum mechanics and induced beam theories, Lecture Notes in Applied and Computational Mechanics 75, Springer, 2015.

[Eugster 2015b] S. R. Eugster, "An intrinsic geometric formulation of the equilibrium equations in continuum mechanics", Proc. Appl. Math. Mech. 15:1 (2015), 289-290.

[Eugster and dell'Isola 2017a] S. R. Eugster and F. dell'Isola, "Exegesis of the introduction and Sect. I from "Fundamentals of the mechanics of continua" by E. Hellinger", Z. Angew. Math. Mech. 97:4 (2017), 477-506.

[Eugster and dell'Isola 2017b] S. R. Eugster and F. dell'Isola, "Exegesis of Sect. II and III.A from "Fundamentals of the mechanics of continua" by E. Hellinger", Z. Angew. Math. Mech. (online publication July 2017).

[Eugster and dell'Isola 2017c] S. R. Eugster and F. dell'Isola, "Exegesis of Sect. III.B from "Fundamentals of the mechanics of continua" by E. Hellinger", Z. Angew. Math. Mech. (online publication September 2017).

[Germain 1972] P. Germain, "Sur l'application de la méthode des puissances virtuelles en mécanique des milieux continus”, C. R. Acad. Sci. Paris 274 (1972), A1051-A1055.

[Germain 1973a] P. Germain, "La méthode des puissances virtuelles en mécanique des milieux continus, I: Théorie du second gradient”, J. Mécanique 12 (1973), 235-274.

[Germain 1973b] P. Germain, "The method of virtual power in continuum mechanics, II: Microstructure”, SIAM J. Appl. Math. 25:3 (1973), 556-575.

[Germain 1986] P. Germain, Mécanique, vol. I, École Polytechnique, 1986. 
[Glocker 2001] C. Glocker, Set-valued force laws: dynamics of non-smooth systems, Lecture Notes in Applied Mechanics 1, Springer, 2001.

[Green and Zerna 1954] A. E. Green and W. Zerna, Theoretical elasticity, Oxford University, 1954.

[Gurtin 1981] M. E. Gurtin, An introduction to continuum mechanics, Mathematics in Science and Engineering 158, Academic, 1981.

[Hamel 1912] G. Hamel, Elemetare Mechanik: ein Lehrbuch, Teubner, 1912.

[Hamel 1927] G. Hamel, "Die Axiome der Mechanik", Kapitel 1, pp. 1-42 in Grundlagen der Mechanik: Mechanik der Punkte und starren Körper, edited by R. Grammel, Handbuch der Physik V, Springer, 1927.

[Hamel 1967] G. Hamel, Theoretische Mechanik: eine einheitliche Einführung in die gesamte Mechanik, Grundlehren der Mathematischen Wissenschaften 57, Springer, 1967.

[Haupt 2002] P. Haupt, Continuum mechanics and theory of materials, 2nd ed., Springer, 2002.

[Hellinger 1914] E. Hellinger, "Die allgemeinen Ansätze der Mechanik der Kontinua”, pp. 602-694 in Enzyklopädie der Mathematischen Wissenschaften mit Einschluss ihrer Anwendungen, vol. IV-4: Mechanik, edited by F. Klein and C. Müller, Teubner, 1914.

[Hilbert 1901] D. Hilbert, "Mathematische Probleme", Arch. Math. Phys. 3:1 (1901), 44-63, 213237.

[Hilbert 1902] D. Hilbert, "Mathematical problems", Bull. Amer. Math. Soc. 8:10 (1902), 437-479.

[Holzapfel 2000] G. A. Holzapfel, Nonlinear solid mechanics: a continuum approach for engineering, Wiley, 2000.

[Kirchhoff 1876] G. Kirchhoff, Vorlesungen über mathematische Physik, Teubner, 1876.

[Kühnel 2013] W. Kühnel, Differentialgeometrie: Kurven - Flächen - Mannigfaltigkeiten, 6th ed., Springer, 2013.

[Lagrange 1788] J.-L. Lagrange, Méchanique analytique, Desaint, 1788.

[Lamé 1852] G. Lamé, Leçons sur la théorie mathématique de l'élasticité des corps solides, École Polytechnique, 1852.

[Lee 2013] J. M. Lee, Introduction to smooth manifolds, 2nd ed., Graduate Texts in Mathematics 218, Springer, 2013.

[Lemaitre and Chaboche 1990] J. Lemaitre and J.-L. Chaboche, Mechanics of solid materials, Cambridge University, 1990.

[Liu 2002] I.-S. Liu, Continuum mechanics, Springer, 2002.

[Macvean 1968] D. B. Macvean, "Die Elementararbeit in einem Kontinuum und die Zuordnung von Spannungs- und Verzerrungstensoren”, Z. Angew. Math. Phys. 19:2 (1968), 157-185.

[Malvern 1965] L. E. Malvern, Introduction to the mechanics of a continuous medium, Prentice-Hall, 1965.

[Mariano and Galano 2015] P. M. Mariano and L. Galano, Fundamentals of the mechanics of solids, Springer, 2015.

[Maugin 1980] G. A. Maugin, "The method of virtual power in continuum mechanics: application to coupled fields", Acta Mech. 35:1-2 (1980), 1-70.

[Müller and Timpe 1914] C. H. Müller and A. Timpe, "Die Grundgleichungen der mathematische Elastizitätstheorie", pp. 1-54 in Enzyklopädie der Mathematischen Wissenschaften mit Einschluss ihrer Anwendungen, vol. IV-4: Mechanik, edited by F. Klein and C. Müller, Teubner, 1914.

[Munkres 2000] J. R. Munkres, Topology, 2nd ed., Prenctice Hall, 2000. 
[Murnaghan 1937] F. D. Murnaghan, "Finite deformations of an elastic solid", Amer. J. Math. 59:2 (1937), 235-260.

[Noll 1959] W. Noll, "The foundations of classical mechanics in the light of recent advances in continuum mechanics", pp. 266-281 in The axiomatic method: with special reference to geometry and physics (Berkeley, CA, 1957-1958), edited by L. Henkin et al., North-Holland, 1959.

[Noll 1974] W. Noll, "La mécanique classique, basée sur un axiome d'objectivité", pp. 135-144 in The foundations of mechanics and thermodynamics, Springer, 1974.

[Ogden 1997] R. W. Ogden, Nonlinear elastic deformations, Dover, 1997.

[Piola 1832] G. Piola, "La meccanica de' corpi naturalmente estesi: trattata col calcolo delle variazioni”, pp. 201-236 in Opuscoli matematici e fisici di diversi autori, vol. I, Giusti, 1832.

[Piola 1848] G. Piola, "Intorno alle equazioni fondamentali del movimento di corpi qualsivogliono, considerati secondo la naturale loro forma e costituzione", pp. 1-186 in Memorie di matematica e fisica della Società italiana delle scienze, vol. XXIV-1, Camera, 1848.

[Piola 2014] G. Piola, The complete works of Gabrio Piola, vol. I, edited by F. dell'Isola et al., Advanced Structured Materials 38, Springer, 2014.

[Poisson 1829] M. Poisson, "Mémoire sur l'équilibre et le mouvement des corps élastique", Mém. Acad. Roy. Sci. 8 (1829), 357-570.

[Salençon 2000] J. Salençon, Mécanique des milieux continus, vol. I: Concepts généraux, École Polytechnique, 2000.

[Salençon 2001] J. Salençon, Handbook of continuum mechanics: general concepts, thermoelasticity, Springer, 2001.

[Schwartz 1951] L. Schwartz, Théorie des distributions, vol. II, Publications de l'Institut de Mathématique de l’Université de Strasbourg 10, Hermann, 1951.

[Sedov 1972] L. I. Sedov, A Course in Continuum Mechanics, vol. 4: Basic equations and analytical techniques, Wolters-Noordhoff, 1972.

[Segev 1986] R. Segev, "Forces and the existence of stresses in invariant continuum mechanics", $J$. Math. Phys. 27:1 (1986), 163-170.

[Spencer 2004] A. J. M. Spencer, Continuum mechanics, Dover, 2004.

[Strichartz 1994] R. S. Strichartz, A guide to distribution theory and Fourier transforms, CRC, 1994.

[Thomson and Tait 1867] W. Thomson and P. G. Tait, "Abstract dynamics", division II, pp. 438-849 in Treatise on natural philosophy, vol. 1, Oxford University, 1867.

[Timoshenko and Goodier 1951] S. Timoshenko and J. N. Goodier, Theory of Elasticity, 2nd ed., McGraw-Hill, 1951.

[Toupin 1964] R. A. Toupin, “Theories of elasticity with couple-stress", Arch. Rational Mech. Anal. 17 (1964), 85-112.

[Truesdell 1991] C. A. Truesdell, A first course in rational continuum mechanics, vol. 1: General concepts, 2nd ed., Pure and Applied Mathematics 71, Academic, 1991.

[Truesdell and Noll 1965] C. Truesdell and W. Noll, The non-linear field theories of mechanics, Handbuch der Physik III-3, Springer, 1965.

[Truesdell and Toupin 1960] C. Truesdell and R. Toupin, The classical field theories, Handbuch der Physik III-1, Springer, 1960.

[Volokh 2006] K. Y. Volokh, "Lagrangian equilibrium equations in cylindrical and spherical coordinates", Comput. Mater. Con. 3:1 (2006), 37-42. 
Received 18 Nov 2016. Revised 22 May 2017. Accepted 19 Aug 2017.

SIMON R. EUGSTER: eugster@inm.uni-stuttgart.de Institute for Nonlinear Mechanics, University of Stuttgart, Stuttgart, Germany CHRISTOPH GLOCKER: glocker@imes.mavt.ethz.ch ETH Zürich, Zurich, Switzerland 
EDITORIAL BOARD

ANTONIO CARCATERRA

ERIC A. CARLEN

FRANCESCO DELL'ISOLA

RAFFAELE ESPOSITO

ALBERT FANNJIANG

Gilles A. FranCFORT

Pierangelo MARCATI

JEAN-JACQUES MARIGO

PETER A. MARKOWICH

MARTIN OSTOJA-STARZEWSKI

PIERRE SEPPECHER

DAVID J. STEIGMANN

PAUl STEINMANN

PierRe M. SuQueT

MANAGING EDITORS

MICOL AMAR

CORRADO LATTANZIO

ANGELA MADEO

MARTIN OSTOJA-STARZEWSKI

\section{ADVISORY BOARD}

ADNAN AKAY

Holm AltenBaCH

MICOL AMAR

HARM ASKES

TEODOR ATANACKOVIĆ

VICTOR BERDICHEVSKY

GUY BOUCHITTÉ

ANDREA BRAIDES

ROBERTO CAMASSA

MAURO CARFORE

ERIC DARVE

FELIX DARVE

ANNA DE MASI

GianPiEtro DEL Piero

EMMANUELE Di BENEDETTO

BERNOLD FIEDLER

IRENE M. GAMBA

DAVID Y. GAO

SERGEY GAVRILYUK

TIMOTHY J. HEALEY

DOMINIQUE JEULIN

ROGER E. KHAYAT

CORRADO LATTANZIO

ROBERT P. LIPTON

ANGELO LUONGO

ANGELA MADEO

JUAN J. MANFREDI

CARLO MARCHIORO

GÉRARD A. MAUGIN

ROBERTO NATALINI PATRIZIO NEFF

ANDREY PIATNITSKI

ERRICO PRESUTTI

MARIO PULVIRENTI

LUCIO RUSSO

Miguel A. F. SANJUAN

PATRICK SElvadurai

ALEXANDER P. SEYRANIAN

MIROSLAV ŠILHAVÝ

GUIDO SWEERS

ANTOINETTE TORDESILLAS

LEV TRUSKINOVSKY

JUAN J. L. VELÁZQUEZ VINCENZO VESPRI ANGELO VULPIANI msp.org/memocs

Università di Roma “La Sapienza”, Italia

Rutgers University, USA

(CO-CHAIR) Università di Roma "La Sapienza", Italia

(TREASURER) Università dell'Aquila, Italia

University of California at Davis, USA

(CO-CHAIR) Université Paris-Nord, France

Università dell'Aquila, Italy

École Polytechnique, France

DAMTP Cambridge, UK, and University of Vienna, Austria

(CHAIR MANAGING EDITOR) Univ. of Illinois at Urbana-Champaign, USA

Université du Sud Toulon-Var, France

University of California at Berkeley, USA

Universität Erlangen-Nürnberg, Germany

LMA CNRS Marseille, France

Università di Roma "La Sapienza", Italia

Università dell'Aquila, Italy

Université de Lyon-INSA (Institut National des Sciences Appliquées), France

(CHAIR MANAGING EDITOR) Univ. of Illinois at Urbana-Champaign, USA

Carnegie Mellon University, USA, and Bilkent University, Turkey

Otto-von-Guericke-Universität Magdeburg, Germany

Università di Roma "La Sapienza", Italia

University of Sheffield, UK

University of Novi Sad, Serbia

Wayne State University, USA

Université du Sud Toulon-Var, France

Università di Roma Tor Vergata, Italia

University of North Carolina at Chapel Hill, USA

Università di Pavia, Italia

Stanford University, USA

Institut Polytechnique de Grenoble, France

Università dell'Aquila, Italia

Università di Ferrara and International Research Center MEMOCS, Italia

Vanderbilt University, USA

Freie Universität Berlin, Germany

University of Texas at Austin, USA

Federation University and Australian National University, Australia

Université Aix-Marseille, France

Cornell University, USA

École des Mines, France

University of Western Ontario, Canada

Università dell' Aquila, Italy

Louisiana State University, USA

Università dell'Aquila, Italia

Université de Lyon-INSA (Institut National des Sciences Appliquées), France University of Pittsburgh, USA

Università di Roma "La Sapienza”, Italia

Université Paris VI, France

Istituto per le Applicazioni del Calcolo "M. Picone", Italy

Universität Duisburg-Essen, Germany

Narvik University College, Norway, Russia

Università di Roma Tor Vergata, Italy

Università di Roma "La Sapienza”, Italia

Università di Roma “Tor Vergata”, Italia

Universidad Rey Juan Carlos, Madrid, Spain

McGill University, Canada

Moscow State Lomonosov University, Russia

Academy of Sciences of the Czech Republic

Universität zu Köln, Germany

University of Melbourne, Australia

École Polytechnique, France

Bonn University, Germany

Università di Firenze, Italia

Università di Roma La Sapienza, Italia

MEMOCS (ISSN 2325-3444 electronic, 2326-7186 printed) is a journal of the International Research Center for the Mathematics and Mechanics of Complex Systems at the Università dell'Aquila, Italy.

Cover image: "Tangle” by $\odot$ John Horigan; produced using the Context Free program (contextfreeart.org).

PUBLISHED BY

7 mathematical sciences publishers

nonprofit scientific publishing

http://msp.org/

(C) 2017 Mathematical Sciences Publishers 
Mathematics and Mechanics of Complex Systems vol. 5 no. 3-4

Forms of the dissipation function for a class of viscoplastic 217 models

Massimo Cuomo

Tumor growth modelling by cellular automata

Ruben Interian, Reinaldo Rodríguez-Ramos, Fernando

Valdés-Ravelo, Ariel Ramírez-Torres, Celso C. Ribeiro and Aura Conci

A variational formulation for fuzzy analysis in continuum 261 mechanics

Rolf Mahnken

On the notion of stress in classical continuum mechanics

Simon R. Eugster and Christoph Glocker

MEMOCS is a journal of the International Research Center for the Mathematics and Mechanics of Complex Systems at the Università dell' Aquila, Italy.

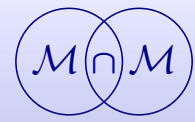

